\title{
Half-metallic ferromagnetism in substitutionally doped boronitrene
}

\author{
A. M. Ukpong ${ }^{1, *}$ and N. Chetty ${ }^{1,2}$ \\ ${ }^{1}$ Department of Physics, University of Pretoria, Hatfield 0002, South Africa \\ ${ }^{2}$ National Institute for Theoretical Physics, Johannesburg 2000, South Africa \\ *E-mail: aniekan.ukpong@up.ac.za
}

We perform first principles molecular dynamics simulations to investigate the magnetoelectronic response of substitutionally-doped boronitrene to thermal excitation. We show that the local geometry, size and edge-termination of the substitutional complexes of boron, carbon or nitrogen determine the thermodynamic stability of the monolayer. We find that hexagonal boron or triangular carbon clusters induce finite magnetic moments with $100 \%$ spinpolarized Fermi-level electrons in boronitrene. In such carbon substitutions, the spontaneous magnetic moment increases with the size of the embedded carbon cluster, and results in halfmetallic ferrimagnetism above $750 \mathrm{~K}$ with a corresponding Curie point of $1250 \mathrm{~K}$, above which the magnetization density vanishes. We predict an ultra-high temperature half-metallic ferromagnetic phase in impurity-free boronitrene, when any three nearest neighbour nitrogen atoms are substituted with boron, with unquenched magnetic moment up to its melting point.

PACS numbers: 71.15.Nc, 71.55.Ht, 73.22.Pr

\section{INTRODUCTION}

The search for spintronic effects in intrinsically nonmagnetic materials is attracting current interests $^{1-4}$ due to promising new functionalities ${ }^{5,6}$ in microelectronics. To this extent, the discovery of metal-free magnetism in carbon-only materials, ${ }^{7,8}$ has stimulated efforts to unravel its origin. Nevertheless, although structural defects, ${ }^{9-14}$ itinerant ferromagnetism, ${ }^{15,16}$ and negatively-curved $s p^{2}$-bonded nanoregions, ${ }^{17}$ have been suggested as possible sources of the spontaneous magnetic moment in carbon-based materials, current spintronic devices still rely on materials with $d$ - and $f$-electron magnetism. In order to facilitate the realisation of nanoscale spintronics, it is important to predict ways of controlling the magnetism in graphenic nanostructures, in order to enhance their potentials for technological applications, and to overcome the well-known ${ }^{18}$ shortcomings of current technologies.

The ballistic electron transport in graphene means that conduction electrons travel through long mean-free paths due to the combined effect of low hyperfine interactions and small spinorbit coupling. These result in exotic properties such as giant magnetoresistance, ${ }^{19}$ and low resistance to current flow due to minimal scattering of delocalised electrons with ion cores. Consider that boronitrene, a one-atom-thick plane of hexagonal boron nitride $(h-\mathrm{BN}),{ }^{20}$ is isostructural to graphene. ${ }^{21}$ Hybrid $\mathrm{BCN}$ nanomaterials consisting of embedded graphene flakes in boronitrene can now be successfully synthesized ${ }^{22,23}$ using thermal catalytic chemical vapour deposition method. Such monolayers possess tunable magneto-electronic properties that depend on the relative concentrations of the constituent species. ${ }^{24-27}$ First principles calculations, based on density functional theory, show that the ordered magnetic moments can be induced in such hybrid BCN nanolayers by doping with transition metal atoms, ${ }^{28}$ adsorption of non-metal impurities, ${ }^{29}$ or by introduction of vacancies and antisites. 10-15,30-33 $^{-10}$

Following the successful demonstration of the in situ electron-beam-assisted postsynthetic 
doping of boron nitride nanostructures with carbon, ${ }^{34-36}$ Berseneva et al. ${ }^{37}$ showed, via extensive $a b$ initio calculations, that although the structural response of the $h$-BN nanostructures to radiation damage is relevant, the mechanism of the postsynthetic doping with carbon is governed by the energetics of the charged state of the nanoinclusions. As a result, they suggested the use of spatially-localised electron-beam irradiation for making the carbon islands and nanoribbons for embedding into $h$-BN nanosheets. In addition, their charged-defects calculations demonstrate the possibility of tuning the induced magnetic moments via the charge state. ${ }^{37}$ Nevertheless, despite improved band gap, and better descriptions of the magnetic exchange couplings in the $h$-BN monolayer, the dependence of the defect-induced magnetic moments on the charge state of the $h$ $\mathrm{BN}$ monolayer is still not clear. We show here that apart from the magnetic moment, the kind of magnetic coupling can also be tuned by varying the charge-injection levels.

The electronic structure in $h$-BN nanostructures is significantly modified by changing the doping level either through electron or hole injection. Heavy doping with donor or acceptor atoms gives significantly different Fermi levels compared to the undoped material. The prediction of the thermodynamic stability of heavily hole- or electron-doped boron nitride nanomaterials, and the characterisation of their associated magnetic couplings could open up possible areas of applications in nansocale magnetoelectronics. Hence, in addition to the substitutional carbon antisite clusters, which are known ${ }^{37}$ to carry charge-tunable local magnetic moments, we consider also the effects of intrinsic antisite clusters as non-metallic dopants in the $h$-BN monolayer. Considering that the edge-localized defect states, ${ }^{11}$ which induce half-metallicity in such hybrid BCN nanoribbons ${ }^{28,33,33,38}$ only occur in zigzag-edged graphene nanoribbons, ${ }^{39-41}$ and not in armchair-edged graphene nanoribbons, we investigate the effects of the local geometry of embedded graphene flakes on the electronic structure to unravel the origin of the half-metallic electronic structure.

In this paper, we investigate the response of the magnetoelectronic properties of substitutionally-doped $h$-BN monolayers to thermal excitation. In particular, the possibility of realizing half-metallic ferrimagnetism with zero magnetization in $h$-BN nanostructures is explored. We consider the properties of two classes of substitutional defect complexes. The 'type $\mathrm{A}^{\prime}$ complexes are formed from the binding of substitutional defects of $\mathrm{B}, \mathrm{C}$, and $\mathrm{N}$ atoms on nearest neighbour positions in monolayer $h$-BN. It is also shown that the ordered magnetic moment, which are induced in $h$-BN nanosheets by type A substitutional complexes are tunable by electron or hole injection. We also investigate the effect of different edge-termination geometries on the electronic properties of hybrid monolayers that contain self-assembled clusters of boron, carbon or nitrogen (hereinafter denoted as 'type B' complexes). We find that an ultrahigh temperature half-metallic ferromagnetic phase is obtained when boron substitutes any three near neighbour nitrogen atoms in boronitrene.

\section{COMPUTATIONAL METHODOLOGY}

Substitutionally-doped monolayers of $h$-BN are modelled in the periodic supercell geometry in order to exploit the plane wave formulation of the electronic structure problem. Periodic images of the layers are separated with a vacuum region of height $15 \AA$ to avoid spurious interlayer interactions. Spin-polarised electronic structure calculations were performed using the Vienna $a b$ initio simulation package (VASP), ${ }^{42-45}$ as implemented in the MedeA suite. ${ }^{46}$ The exchange-correlation potential was described in the generalized gradient approximation, as parameterized by Perdew, Burke, and Ernzerhof (PBE). ${ }^{47}$ The interactions between valence electrons and ion cores are described using projector-augmented wave (PAW) potentials. ${ }^{48,49} \mathrm{~A}$ cut-off limit of $500 \mathrm{eV}$ was set for kinetic energy expansion in the plane wave basis. The total energy was converged to within $10^{-7} \mathrm{eV}$. In the self-consistent calculations of electronic energies, electron states were populated using the Methfessel-Paxton scheme, ${ }^{50}$ with a smearing width of 
$0.4 \mathrm{eV}$. The Brillouin zone was sampled using the Monkhorst-Pack ${ }^{51}$ grid of size $10 \times 10 \times 1$.

The monolayers were first obtained by simulated doping of pristine $h$-BN monolayer with type A complexes. The single-atom carbon substitutions (i.e. $C_{B}$ and $C_{N}$ ) have the lowest formation energy, ${ }^{52}$ and can also induce half-metallicity in $h$-BN nanostructures. ${ }^{33}$ We therefore consider $h$-BN monolayers that are doped with type $\mathrm{B}$ complexes. The atomic species that terminate the edges of type B complexes control the postsynthetic substitution of boron and nitrogen atoms with carbon in electron beam irradiated $h$-BN nanostructures. ${ }^{35-37}$ In the charged defect calculations, a uniform background charge is added to maintain the global charge neutrality of the supercell. ${ }^{53}$ The formation energy $E_{f}^{q}(D)$ of a defect in charge state $q$ was calculated in the Zhang-Northrup formalism ${ }^{54}$ as $E_{f}^{q}(D)=E_{\text {total }}(q, D)-\sum_{i} n_{i} \mu_{i}+q\left(\mu_{e}+\varepsilon_{v}\right)$, where $E_{\text {total }}(q, D)$ is the total energy of the supercell containing a defect $D$ of charge $q$, and $n_{i}$, is the number of species $i$ required to create defect $D$, and $\mu_{i}$ is the corresponding atomic chemical potential, and $\mu_{e}$ is electron chemical potential relative to the valence band maximum (VBM, denoted as $\varepsilon_{v}$ ) of the pristine $h$-BN monolayer. The transition energy level $\varepsilon\left(q / q^{\prime}\right)$ of defects was obtained as $\varepsilon\left(q / q^{\prime}\right)=\left[E_{f}^{q}(D)-E_{f}^{q^{\prime}}(D)\right] /\left(q^{\prime}-q\right)$. This corresponds to the Fermi energy at which the formation energy $E_{f}^{q}(D)$ of defect $D$ with charge $q$ equals the formation energy of the same defect $E_{f}^{q^{\prime}}(D)$ when it carries charge $q^{\prime}$.

Large quasiparticle corrections apply to the band gap of boronitrene. ${ }^{52,55}$ Hence, the range in which the electron chemical potential varies during charge injection is influenced by such corrections. Nevertheless, the electronic structure of boronitrene is known ${ }^{55}$ to give an improved band gap and a better description of exchange coupling, when the hybrid functional of Heyd, Scuseria, and Ernzerhof (HSE06) is used. ${ }^{56,57}$ It has been shown ${ }^{37}$ that the formation energies obtained using PBE and HSE functionals agree quite well, when the electron chemical potential is re-scaled using the ratio of the band gaps obtained using the PBE and HSE approximations for the $\mathrm{XC}$ potential. Due to its good convergence of total energy with respect to $k$-point sampling, the HSE06 hybrid functional was used to calculate the magnetic moment induced by the charge states of the smallest size of type B complexes whose ground states exhibit the half-metallic electronic structure.

The response of the magnetoelectronic properties of the hybrid $\mathrm{BCN}$ monolayers to heat was also investigated using ab initio molecular dynamics simulations. The simulations are performed in the canonical ensemble averaging scheme, where the number of atoms, volume and temperature (NVT) are always kept constant. Each hybrid monolayer was directly equilibrated at a given final temperature $T_{f}$ such that $0 \leq T_{f}(\mathrm{~K}) \leq 2000$. Atomic positions in all equilibrated model structures were fully relaxed, using the conjugate gradient algorithm, until the average force on an atom is smaller than $2 \mathrm{meV} / \AA$. The maximum temperature considered in this study (i.e. $2500 \mathrm{~K}$ ) is far lower than the melting point (i.e. $3400 \pm 20 \mathrm{~K}$ ) of bulk hexagonal boron nitride. ${ }^{58}$ Aas a result, we apply a uniform thermal processing to all the doped monolayers. By equilibrating the layers at $T_{f}$ over a fixed duration of $150 \mathrm{ps}$, the spurious local bonding structures that form when non-uniform thermal gradients are applied ${ }^{59}$ are avoided. Finite-size corrections for charged configurations ${ }^{60-62}$ were analysed by extrapolating the total energies to dilute limits.

\section{RESULTS AND DISCUSSION}

A. Thermodynamic stability

1. Defect formation energies 
Figure 1(a) shows the ball-and-stick models of the unrelaxed local atomic structures in the substitutional complexes considered in the present study. An intrinsic antisite complex is obtained as a hexagonal ring cluster $(\mathrm{HRC})$ of boron $\left(\mathrm{B}_{3 \mathrm{~N}}\right)$ and nitrogen $\left(\mathrm{N}_{3 \mathrm{~B}}\right)$ when three boron or nitrogen atoms substitute the three nearest neighbour nitrogen or boron atoms within a hexagonal ring of boronitrene. Similarly, the carbon impurity antisite complex is obtained as a HRC when six carbon atoms substitute for the three nitrogen and three boron atoms within the hexagonal ring. On the other hand, the triangular ring cluster (TRC) of carbon is obtained when at least four carbon atoms substitute for boron and nitrogen. The smallest TRC of carbon is either the Bterminated $\left(\mathrm{C}_{1 \mathrm{~B}}+\mathrm{C}_{3 \mathrm{~N}}\right)$ or $\mathrm{N}$-terminated $\left(\mathrm{C}_{1 \mathrm{~N}}+\mathrm{C}_{3 \mathrm{~B}}\right)$ star-shaped carbon cluster obtained when the number of substitutional carbon atoms $N_{C}=4$. Larger TRCs are obtainable from the star-shaped cluster for $N_{C}>4$. The interaction between periodic images of defects is known to introduce errors in the total energy. These errors become larger for charged defects. The formation energies were corrected using the Markov-Payne approach. ${ }^{63}$ Using the most extended type-B complexes (i.e. $\mathrm{C}_{6 \mathrm{~B}}+\mathrm{C}_{10 \mathrm{~N}}$ ), the formation energy in $\mathrm{N}$-rich condition was converged to $5.09 \mathrm{eV}$ using defect densities as suggested in Ref. 64. For cells containing up to 100 atoms, the results of the $5 \times 5$ cell presented here, shows a maximum of $\sim 3.2 \%$ difference in formation energy after finite-size corrections.

The $0 \mathrm{~K}$ formation energies of uncharged type A and type B complexes are shown in Fig. 1(b) and (c) respectively, as a function of changes in atomic chemical potential, $\Delta \mu$. The formation energy is thermodynamically constrained to vary within the interval determined by the stability limits of pristine monolayer relative to nitrogen gas $\left(\mathrm{N}_{2}\right)$ and metallic boron $(\alpha-\mathrm{B})$. This range, determined by the enthalpy of formation $\left(\Delta H_{f}=2.6 \mathrm{eV}\right)$, is given by $-2.6 \leq \Delta \mu \leq 2.6$ where the limits $\Delta \mu_{\min }$ and $\Delta \mu_{\max }$ correspond to $\mathrm{N}$-rich and B-rich growth conditions respectively. The nonstoichiometric $\mathrm{B}_{1 \mathrm{~N}}+\mathrm{C}_{1 \mathrm{~B}}$ and $\mathrm{N}_{1 \mathrm{~B}}+\mathrm{C}_{1 \mathrm{~N}}$ complexes present their lowest formation energies in $\mathrm{B}$ rich and N-rich conditions [see Fig. 1(b)], respectively. Formation energy of stoichiometric $\mathrm{B}_{1 \mathrm{~N}}+\mathrm{N}_{1 \mathrm{~B}}$ and $\mathrm{C}_{1 \mathrm{~B}}+\mathrm{C}_{1 \mathrm{~N}}$ complexes is not sensitive to growth conditions; and we obtain $2.48 \mathrm{eV}$ $\left(\mathrm{C}_{1 \mathrm{~B}}+\mathrm{C}_{1 \mathrm{~N}}\right)$ and $7.73 \mathrm{eV}\left(\mathrm{B}_{1 \mathrm{~N}}+\mathrm{N}_{1 \mathrm{~B}}\right)$ in the neutral state. Similarly, the lowest formation energies of $\mathrm{N}_{1 \mathrm{~B}}+\mathrm{C}_{1 \mathrm{~N}}$ and $\mathrm{B}_{1 \mathrm{~N}}+\mathrm{C}_{1 \mathrm{~B}}$ complexes are $4.89 \mathrm{eV}$ (N-rich) and $4.91 \mathrm{eV}$ (B-rich), showing enhanced defect stability in growth environments that correspond to the excess atomic species in the layer.
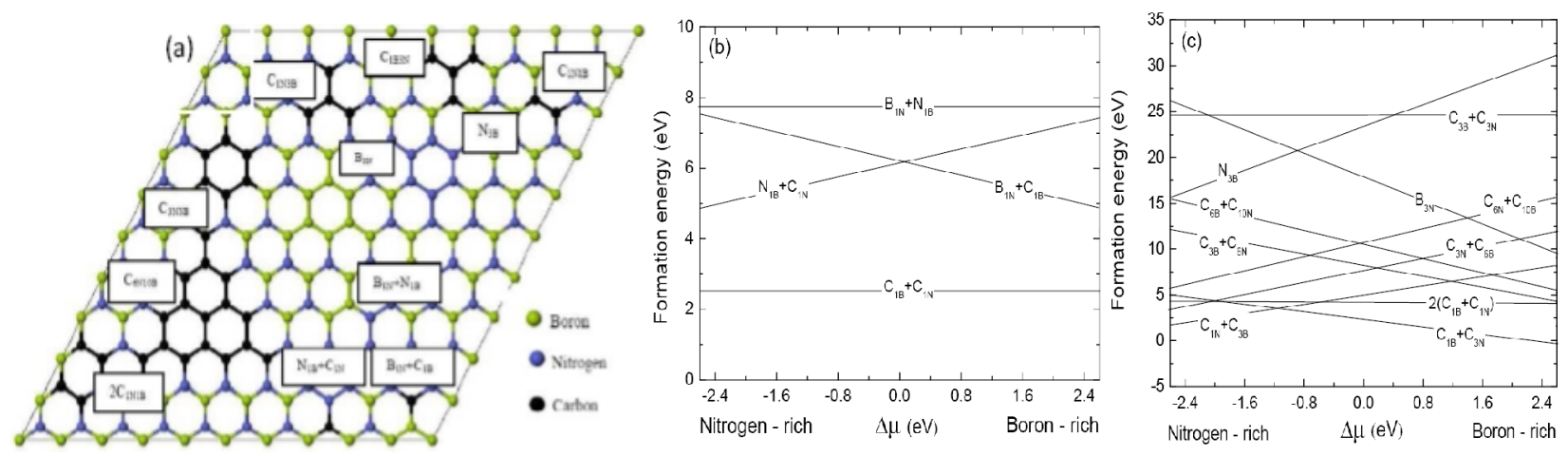

FIG. 1. Local defect structures in hybrid BCN monolayers (a), and formation energy of neutral species of: type A (b), and type B (c) substitutional complexes.

The dependence of the formation energy of uncharged type $\mathrm{B}$ complexes on $\Delta \mu$ is shown in Fig. 1(c). For the star-shaped C-cluster, we find that the B-termination, i.e. $\mathrm{C}_{1 \mathrm{~B}}+\mathrm{C}_{3 \mathrm{~N}}$, is more stable in B-rich condition by $3.29 \mathrm{eV}$ compared to the N-termination of the same complex i.e. $\mathrm{C}_{1 \mathrm{~N}}+\mathrm{C}_{3 \mathrm{~B}}$ complex, in the N-rich condition. The $\mathrm{C}_{3 \mathrm{~B}}+\mathrm{C}_{3 \mathrm{~N}}$ complex is stoichiometric; hence, its formation energy $(24.57 \mathrm{eV})$ is independent of growth conditions. By contrast, the $\mathrm{N}_{3 \mathrm{~B}}$ and $\mathrm{B}_{3 \mathrm{~N}}$ complexes are nonstoichiometric. In the B-rich condition for instance, the $\mathrm{B}_{3 \mathrm{~N}}$ complex presents 
its lowest formation energy of $9.05 \mathrm{eV}$; far higher than the highest formation energy of any typeA complex. In addition, although the lowest formation energy of the $\mathrm{N}_{3 \mathrm{~B}}$ complex $(15.49 \mathrm{eV})$ occurs in N-rich condition, we predict that its formation is energetically unfavourable relative to the most unstable type A complex i.e. $\mathrm{B}_{1 \mathrm{~N}}+\mathrm{N}_{1 \mathrm{~B}}$ [see Fig. 1(b)]. Fig. 1(c) shows significantly reduced stability of $h$-BN monolayers when the HRC of boron, carbon or nitrogen are embedded in the layer compared to introduction of type A complexes. The formation energy of the HRC of $\mathrm{B}, \mathrm{C}$ or $\mathrm{N}$ is one order of magnitude higher than formation energies of the two-atom $\mathrm{C}$-line $\left(\mathrm{C}_{1 \mathrm{~N}}+\mathrm{C}_{1 \mathrm{~B}}\right)$, and the double two-atom $\mathrm{C}$-line $2\left(\mathrm{C}_{1 \mathrm{~N}}+\mathrm{C}_{1 \mathrm{~B}}\right)$. The magnitudes of the formation energies of the $\mathrm{C}_{1 \mathrm{~N}}+\mathrm{C}_{1 \mathrm{~B}}, 2\left(\mathrm{C}_{1 \mathrm{~N}}+\mathrm{C}_{1 \mathrm{~B}}\right), \mathrm{C}_{1 \mathrm{~N}}+\mathrm{C}_{3 \mathrm{~B}}$ and $\mathrm{C}_{1 \mathrm{~B}}+\mathrm{C}_{3 \mathrm{~N}}$ complexes are comparable to those of single-atom carbon substitutions on the $\mathrm{B}$ (i.e. $\mathrm{C}_{\mathrm{B}}$ ) and $\mathrm{N}$ (i.e. $\mathrm{C}_{\mathrm{N}}$ ) sublattices. ${ }^{52}$ Recently, Berseneva et $a l .{ }^{37}$ showed that the stability of carbon substitutional defects in the hybrid BCN layer is sensitive to the number $\left(N_{\mathrm{C}}\right)$ of $\mathrm{C}$ atoms. The spontaneous formation of $\mathrm{B}$ or $\mathrm{N}$ clusters in monolayer $h$-BN is energetically not favourable because of the high energy penalties required [see Fig. 1(c)]. Hence, we will only investigate the dependence of the formation energy of complexes on the number of substituted $\mathrm{B}\left(N_{\mathrm{B}}\right)$ and $\mathrm{N}\left(N_{\mathrm{N}}\right)$ atoms when carbon clusters (i.e. graphene flakes) are embedded in the $h$-BN monolayer. In this case, $N_{\mathrm{C}}=N_{\mathrm{B}}+N_{\mathrm{N}}$ for the carbon substitutions, and the limit $N_{\mathrm{C}}=50$ corresponds to $5 \times 5$ supercell model of graphene.

The formation energies of type B complexes are sensitive to the type of atoms that terminated the edges of the complexes, and the number $N_{C}$ of substituted atoms. In single species edge-terminations, we find that the formation energy increases (or decreases) in the growth condition that correspond to the highest (or lowest) number of substituted atomic species. For instance, although $N_{\mathrm{C}}=4$ in both $\mathrm{C}_{1 \mathrm{~N}}+\mathrm{C}_{3 \mathrm{~B}}$ and $\mathrm{C}_{1 \mathrm{~B}}+\mathrm{C}_{3 \mathrm{~N}}$ complexes, the B-terminated $\mathrm{C}_{1 \mathrm{~B}}+\mathrm{C}_{3 \mathrm{~N}}$ complex is more stable in B-rich condition than in the N-rich condition. Similarly, in the Nterminated analogue, the formation energy in $\mathrm{N}$-rich condition $(2.98 \mathrm{eV})$ is lower than the corresponding formation energy in B-rich condition $(8.16 \mathrm{eV})$. The same dependence of defect stability on edge-termination is also obtained in the $\mathrm{C}_{10 \mathrm{~B}}+\mathrm{C}_{6 \mathrm{~N}}$ and $\mathrm{C}_{6 \mathrm{~B}}+\mathrm{C}_{10 \mathrm{~N}}$ complexes where $N_{\mathrm{C}}$ $=16$, in good agreement with Pruneda. ${ }^{31}$ The scaling of the formation energy with the number of carbon atoms $N_{C}$ in the uncharged $h$-BN monolayers that contain carbon TRCs shows that marginal increases in carbon cluster size results in substantial increases in the formation energy. These correspond to the carbon chemical potential of $-9.22 \mathrm{eV}$, obtained as the ratio of the ground state total energy to the number of carbon atoms in the $5 \times 5$ supercell of pristine graphene. Fig. 1 (c) shows that boron nitride monolayers that contain intrinsic antisite clusters - $\mathrm{B}_{3 \mathrm{~N}}$ or $\mathrm{N}_{3 \mathrm{~B}}$ - are comparatively less stable compared to the monolayers that contain the carbon TRC. In both Brich and N-rich condition, the hybrid $\mathrm{BCN}$ layer shows enhanced stability when the edges of the embedded carbon TRC are terminated with $\mathrm{N}$ and $\mathrm{B}$ atoms respectively. By contrast, Bterminated carbon TRCs shows higher formation energies in N-rich conditions and vice versa, irrespective of the size of the carbon cluster.

\section{Charge transition levels}

Figure 2(a)-(d) shows the effect of charge injection on the thermodynamic stability of type A complexes. The formation energies are constrained to vary with electron chemical potential $\mu_{e}$ in the range $0 \leq \mu_{e}(\mathrm{eV}) \leq 5.08$ because $\mu_{e}$ is normalized to the band gap of pristine $h$-BN monolayer. ${ }^{53}$ For the non-stoichiometric complexes, only growth conditions that give preferential stabilities are shown. Negatively charged (electron-rich) type A complexes have lower formation energies compared to their corresponding positively-charged states at the same $\mu_{e}$. Similarly, Fig. 3(a)-(d) shows that hole injection in type B complexes results in substantially increased formation energies as $\mu_{e}$ is increased. The same trend is also seen type A complexes albeit with comparatively lower energies. Electron-rich type B complexes are more stable as the Fermi level 
is raised. The higher formation energies observed at high positive charge states in HRCs of $\mathrm{B}, \mathrm{C}$, and $\mathrm{N}$ show that heavy hole-doping drives the $h$-BN monolayers toward energetic instability.

Our results show good qualitative agreement with previous PBE calculations for similar carbon TRCs. For instance, the,+ 0 and - charge states of the boron-terminated $\mathrm{C}_{1 \mathrm{~B}}+\mathrm{C}_{3 \mathrm{~N}}$ complex show similar trends in formation energies when compared with the larger carbon complex $\left(4 \mathrm{C}_{1 \mathrm{~B} 3 \mathrm{~N}}\right)$ studied in Ref. 37 . The negatively charged states of the carbon TRC consistently show enhanced stability as the position of the Fermi level is shifted towards higher electron chemical potential. Under increased doping with electrons, the energetics of electron-rich TRC of carbon is consistent with the mechanism proposed by Berseneva et al. ${ }^{37}$ for electron irradiation-mediated substitutional doping of $h$-BN nanostructures with carbon. However, despite the consistency in the predicted stability trends with levels of charge injection, we observe significant discrepancies in the absolute values of the formations energies between the two PBE calculations of the same complex. Therefore, we can only ascribe the observed discrepancy to the size of the carbon TRC used in our calculations, more so that our predicted formation energies in nitrogen-rich condition are consistent with the predicted trend for formation of the $4 \mathrm{C}_{1 \mathrm{~B} 3 \mathrm{~N}}$ complex in the uncharged state when the Fermi level is alligned to $0 \mathrm{eV}{ }^{37}$ Thus, the observed discrepancy is not necessarily a disagreement between two PBE calculations of the same structure because our carbon complex is four times smaller than that of the $4 \mathrm{C}_{1 \mathrm{~B} 3 \mathrm{~N}}$ complex studied in Ref. 37.
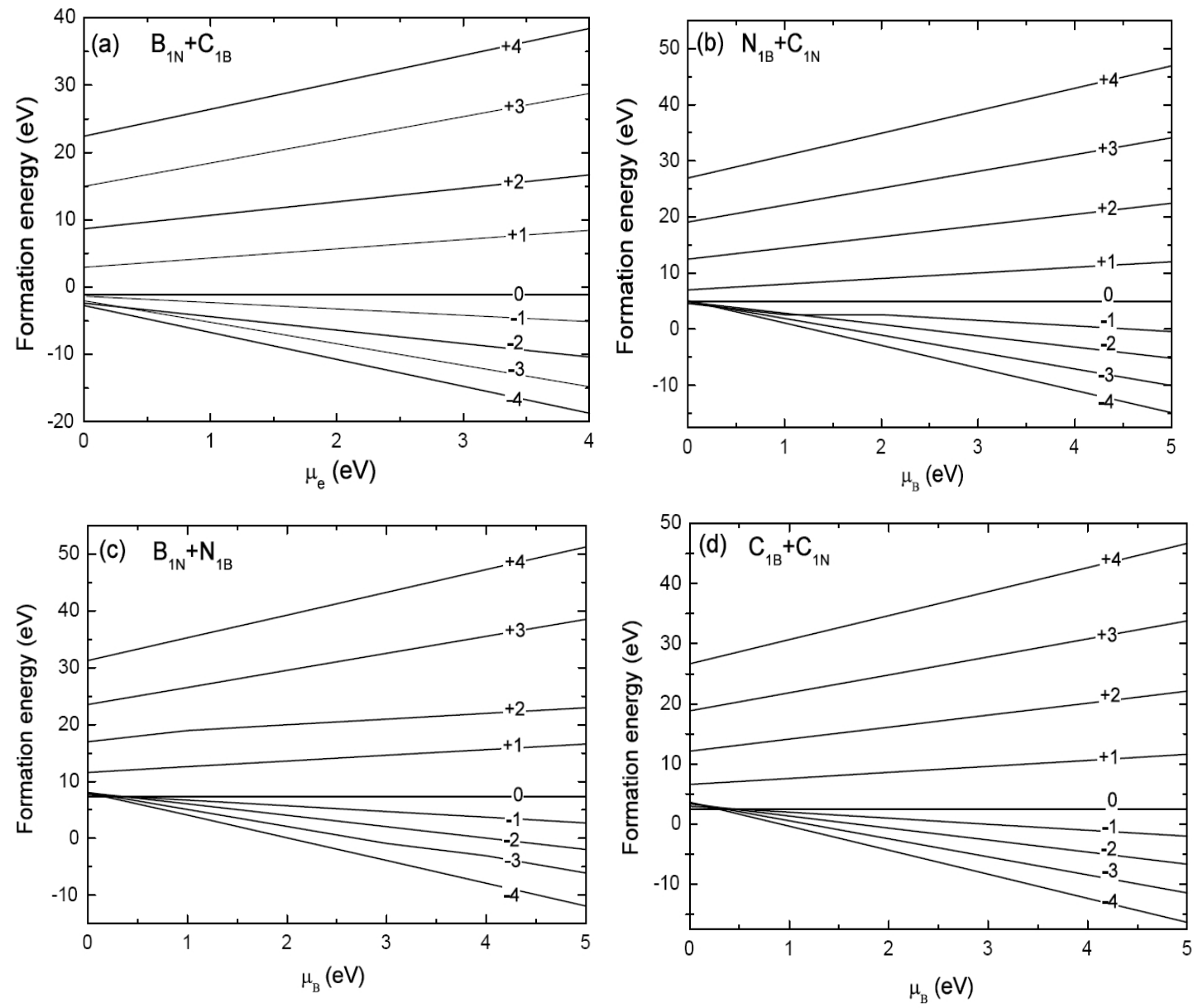

FIG. 2. Lowest formation energies in type $A$ for charged boronitrene containing: $B_{1 N}+C_{1 B}(a)$, $\mathrm{N}_{1 \mathrm{~B}}+\mathrm{C}_{1 \mathrm{~N}}(\mathrm{~b}), \mathrm{B}_{1 \mathrm{~N}}+\mathrm{N}_{1 \mathrm{~B}}(\mathrm{c})$, and $\mathrm{C}_{1 \mathrm{~B}}+\mathrm{C}_{1 \mathrm{~N}}(\mathrm{~d})$. 
Generally, the formation energies of uncharged complexes are constant and independent of changes in the electron chemical potential. The negatively (-) charged complexes show monotonous decrease in formation energy as $\mu_{e}$ increases, whereas positively $(+)$ charged complexes show increased instability at higher $\mu_{e}$. This shows that electron-rich hybrid $h$-BN monolayers may form spontaneously compared to hole-rich layers in good agreement with recent calculations of point defects in monolayer $h$-BN. ${ }^{53}$ The small differences between the formation energies of the complexes suggest that transitions between different charge states of the same complex is not unexpected. Such transition levels are measured in deep level transient spectroscopy (DLTS), and forms the experimental basis for defect identification. ${ }^{65}$ Charges can be injected to give electron-rich (acceptors) or hole-rich (donors) monolayers. We therefore discuss the $0 /+1$ and $0 /-1$ transitions levels of type A substitutional complex. In their neutral states, the $\mathrm{B}_{\mathrm{N}}+\mathrm{C}_{\mathrm{B}}$ complex is an acceptor while $\mathrm{N}_{\mathrm{B}}+\mathrm{C}_{\mathrm{N}}$ complex is a donor relative to pristine $h$ - $\mathrm{BN}$. Similarly, the stoichiometric $\mathrm{B}_{N}+\mathrm{N}_{B}$ and $\mathrm{C}_{\mathrm{B}}+\mathrm{C}_{\mathrm{N}}$ complexes are isoelectronic and contain 200 electrons in the uncharged state, but charges can equally be injected via electron or hole doping. The charge transition levels are calculated relative to the GGA-PBE band edges of pristine $h$-BN when the conduction band maximum $\left(\mathrm{CBM}\right.$, denoted by $\left.\varepsilon_{c}\right)$ is fixed at the $\Gamma$-point. ${ }^{53}$
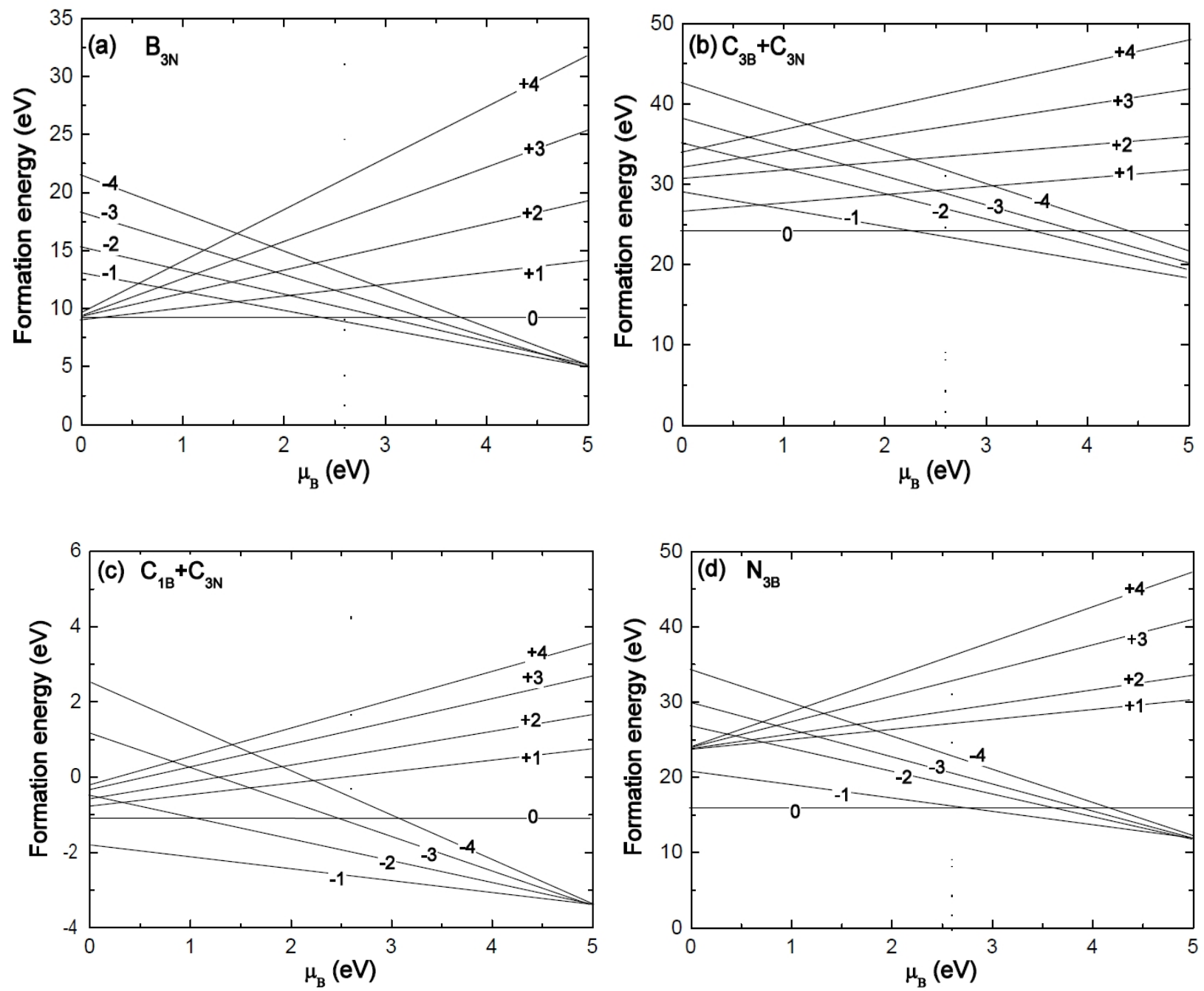

FIG. 3. Lowest formation energies in type $B$ complexes: $B_{3 N}(a), C_{3 B}+C_{3 N}(b), C_{1 B}+C_{3 N}$ (c), and $\mathrm{N}_{3 \mathrm{~B}}(\mathrm{~d})$ as a function of electron chemical potential. 
Table I shows the ionization potential of donors in the $0 /+$ charge transitions, and the electron affinity of acceptors for 0/- charge transitions. Donor levels are evaluated relative to $\varepsilon_{v}$, while the acceptor levels are evaluated relative to $\varepsilon_{c}$, in the pristine $h$-BN monolayer, in each case. We find that donor and acceptor levels of $B_{N}+N_{B}, C_{B}+C_{N}$ complexes show similar characteristic as those of $B_{N}+C_{B}$ and $C_{B}+C_{N}$ complexes. Donor transition levels of both stoichiometric and nonstoichiometric complexes localize deeper into the band gap region as the magnitude of the injected charge increases. The deepest donor is the $\mathrm{C}_{\mathrm{B}}+\mathrm{C}_{\mathrm{N}}$ complex, with a calculated $(0 /+1)$ transition energy of $\varepsilon_{\mathrm{c}}-0.11 \mathrm{eV}$, and the shallowest acceptor is the $\mathrm{B}_{\mathrm{N}}+\mathrm{N}_{\mathrm{B}}$ complex with $0 /+1$ transition energy of $\varepsilon_{\mathrm{v}}+0.02 \mathrm{eV}$. Stoichiometric acceptors behave differently; the shallowest level of the $C_{B}+C_{N}$ complexes is obtained at higher charge states (0/-4). Acceptor levels of the $C_{B}+C_{N}$ complexes are localised at least $2.0 \mathrm{eV}$ above the valence band maximum $\varepsilon_{\mathrm{c}}$. We attribute the opposing acceptor characteristics to the effect of carbon because as more electrons are injected into the system, as electron states become localized at lower energies just above the VBM.

Table I. Ionisation energies of donors (in $\mathrm{eV}$ ) relative to the conduction band minimum and electron affinity of acceptors relative to the valence band maximum of type A complexes.

\begin{tabular}{cccccc}
\hline \hline Species & Transition & $\mathrm{B}_{\mathrm{N}}+\mathrm{N}_{\mathrm{B}}$ & $\mathrm{C}_{\mathrm{B}}+\mathrm{C}_{\mathrm{N}}$ & $\mathrm{B}_{\mathrm{N}}+\mathrm{C}_{\mathrm{B}}$ & $\mathrm{N}_{\mathrm{B}}+\mathrm{C}_{\mathrm{N}}$ \\
\hline \multirow{3}{*}{ Donors } & $0 /+1$ & $\varepsilon_{\mathrm{c}}-0.87$ & $\varepsilon_{\mathrm{c}}-0.11$ & $\varepsilon_{\mathrm{c}}-0.57$ & $\varepsilon_{\mathrm{c}}-0.15$ \\
& $0 /+2$ & $\varepsilon_{\mathrm{c}}-1.62$ & $\varepsilon_{\mathrm{c}}-1.12$ & $\varepsilon_{\mathrm{c}}-2.80$ & $\varepsilon_{\mathrm{c}}-1.72$ \\
& $0 /+3$ & $\varepsilon_{\mathrm{c}}-2.27$ & $\varepsilon_{\mathrm{c}}-2.45$ & $\varepsilon_{\mathrm{c}}-3.44$ & $\varepsilon_{\mathrm{c}}-2.75$ \\
& $0 /+4$ & $\varepsilon_{\mathrm{c}}-3.89$ & $\varepsilon_{\mathrm{c}}-3.05$ & $\varepsilon_{\mathrm{c}}-3.52$ & $\varepsilon_{\mathrm{c}}-4.63$ \\
\hline \multirow{3}{*}{ Acceptors } & $0 /-1$ & $\varepsilon_{\mathrm{v}}+0.02$ & $\varepsilon_{\mathrm{v}}+0.45$ & $\varepsilon_{\mathrm{v}}+0.90$ & $\varepsilon_{\mathrm{v}}+0.29$ \\
& $0 /-2$ & $\varepsilon_{\mathrm{v}}+0.15$ & $\varepsilon_{\mathrm{v}}+0.33$ & $\varepsilon_{\mathrm{V}}+0.61$ & $\varepsilon_{\mathrm{v}}+0.12$ \\
& $0 /-3$ & $\varepsilon_{\mathrm{v}}+0.21$ & $\varepsilon_{\mathrm{v}}+0.25$ & $\varepsilon_{\mathrm{v}}+0.39$ & $\varepsilon_{\mathrm{v}}+0.08$ \\
& $0 /-4$ & $\varepsilon_{\mathrm{v}}+0.47$ & $\varepsilon_{\mathrm{v}}+0.21$ & $\varepsilon_{\mathrm{v}}+0.27$ & $\varepsilon_{\mathrm{v}}+0.06$ \\
\hline
\end{tabular}

\section{B. Electronic properties}

\section{Difference charge density distribution}

The site-resolved difference charge density distribution for spin-up (top panels) and spindown (bottom panels) electrons are shown for type A complexes [see Figure 4(a)-(d)] and type B complexes [see Figure 5(a)-(d)]. The difference charge density, in this case, is the difference between the total charge density in the hybrid layer and the free atom charge density of constituents. In both type A and B complexes, spin-up and spin-down electrons clearly localise with different intensities. The isosurface plots show extremum values, in each case, for spin-up (maxima) and spin-down (minima) electrons. Thus, differences between up and down spin densities give the magnetic polarisation density. The density of spin-up electrons is strongly localised along bond axes. Along the HRC of nitrogen in the $\mathrm{N}_{3 \mathrm{~B}}$ complex, there is zero density of spin up electrons. By contrast, the charge density localisation in the HRC of boron $\left(\mathrm{B}_{3 \mathrm{~N}}\right.$ complex) shows slight distortion away from the bond axes. In particular, the majority carrier charge density is strongly localised in the region that shows significantly reduced B-B-B bond angles within the 
$\mathrm{B}$ ring. Although there is a noticeable increase in the charge density along $\mathrm{C}-\mathrm{C}$ bonds relative to $\mathrm{B}-\mathrm{N}$ bonds away from the cluster, majority carrier densities are also localised along bond axes in the embedded carbon clusters. The dominant trend in majority carrier density localization shows that spin-up electrons determine the ionicity and hybridization of B-N bonds in boronitrene. ${ }^{53}$

The minority carrier density in the $\mathrm{N}_{3 \mathrm{~B}}$ complex is localised at nitrogen atoms. Within the HRC however, electron density at the three $\mathrm{N}$ antisites occupying the original B sublattice show higher electron densities relative to the three unperturbed $\mathrm{N}$ sublattices. By contrast, the $\mathrm{B}_{3 \mathrm{~N}}$ and all carbon complexes show minority carrier densities localised on boron atoms and at hollow sites. Interestingly, there is no spin-down charge density in hollow sites when $\mathrm{N}_{3 \mathrm{~B}}$ complex is embedded in the layer. The spin-down electron density shows gradients at hollow sites. At the centroid of the $\mathrm{B}_{3 \mathrm{~N}}$ complex cluster for instance, minority carrier density is low at hollow sites, but rapidly increases radially outwards. Triangular carbon complexes show a distinctly different behaviour because electron density is strongly localised at hollow sites near the centre of the triangular cluster and reduced radially outwards from the centre. In the hexagonal geometry, minority carrier density is weakly localised at the centre of the hexagonal ring. Away from the cluster, it is delocalised across the layer at equal intensity. We also note the zero electron density from the carbon antisites on $\mathrm{N}$ sublattices [Fig. 5(c)]. In the $\mathrm{N}_{3 \mathrm{~B}}$ complex, variable density of spin-down electrons are localised on the nitrogen atoms of the cluster. Away from the centre of the $\mathrm{N}_{3 \mathrm{~B}}$ complex, the density of minority carrier vanishes. The densities at hollow sites denote itinerant defect states and whereas the localised defect states are centred on atoms. The resolution of spin order in the hybrid BCN layers implies that electron states contribute to the resulting magnetoelectronic properties of the layers.
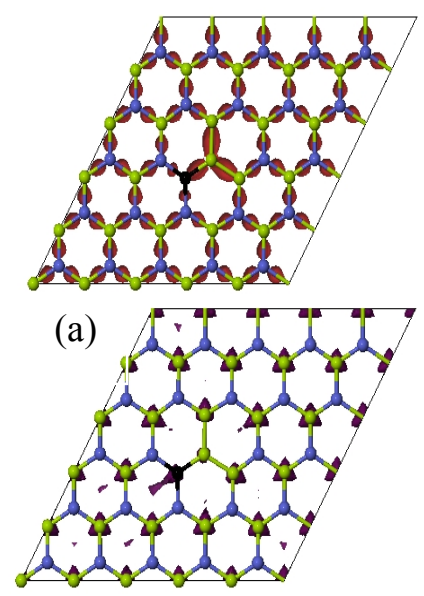

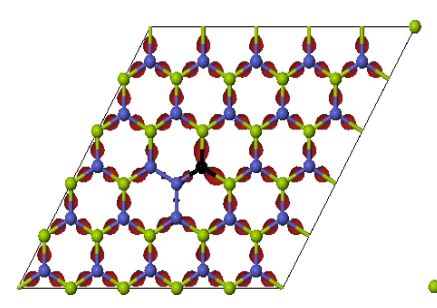

(b)

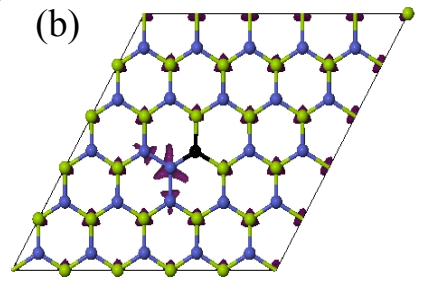

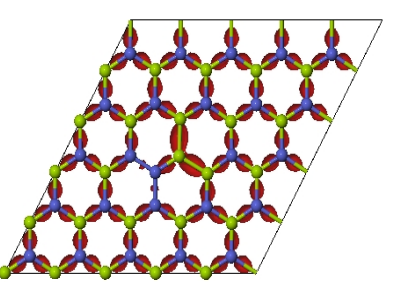

(c)

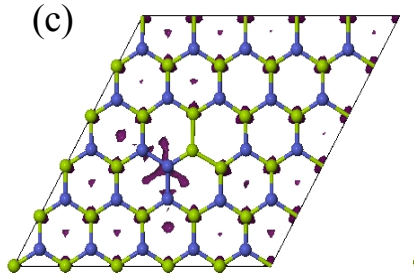

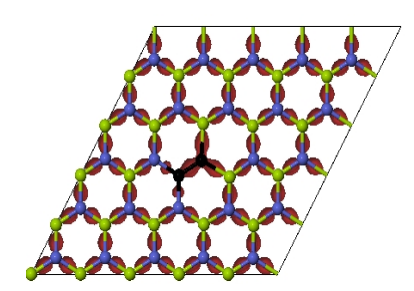

(d)

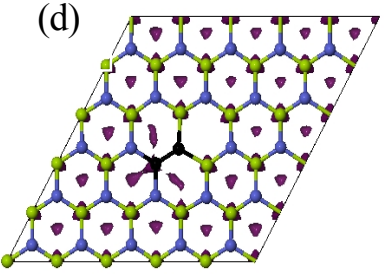

FIG. 4. Site-resolved spin-polarised difference charge densities in boronitrene containing: $\mathrm{B}_{1 \mathrm{~N}}+\mathrm{C}_{1 \mathrm{~B}}(\mathrm{a}), \mathrm{N}_{1 \mathrm{~B}}+\mathrm{C}_{1 \mathrm{~N}}(\mathrm{~b}), \mathrm{B}_{1 \mathrm{~N}}+\mathrm{N}_{1 \mathrm{~B}}(\mathrm{c}), \mathrm{C}_{1 \mathrm{~B}}+\mathrm{C}_{1 \mathrm{~N}}(\mathrm{~d})$ substitutional complexes. 


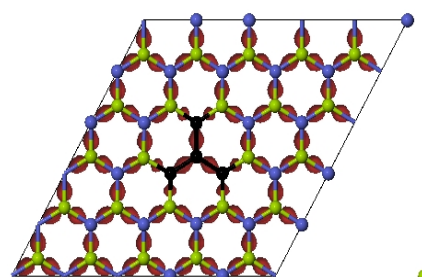

(a)

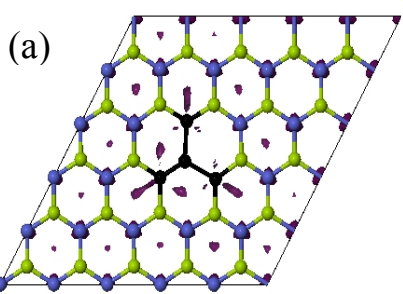

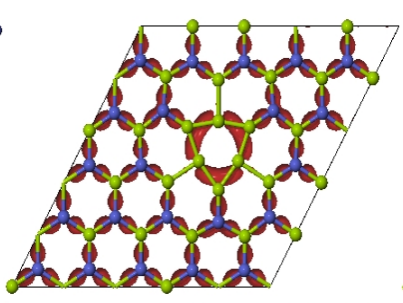

(b)

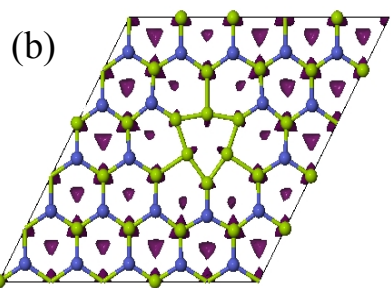

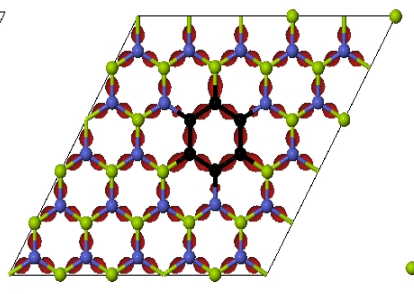

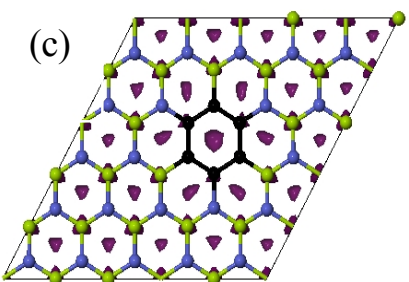

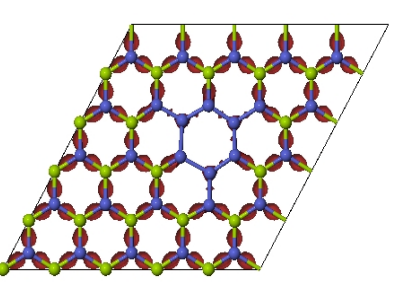

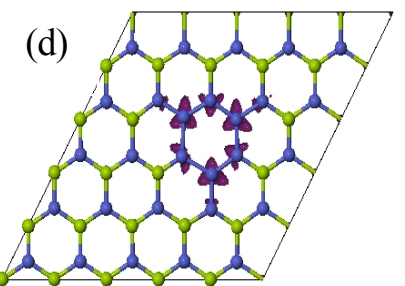

FIG. 5. Site-resolved spin-polarised charge densities in self-assembled substitutional clusters showing: $\mathrm{C}_{1 \mathrm{~B}}+\mathrm{C}_{3 \mathrm{~N}}(\mathrm{a}), \mathrm{B}_{3 \mathrm{~N}}(\mathrm{~b}), \mathrm{C}_{3 \mathrm{~B}}+\mathrm{C}_{3 \mathrm{~N}}(\mathrm{c})$, and $\mathrm{N}_{3 \mathrm{~B}}(\mathrm{~d})$ complexes.

\section{Influence of stoichiometry on spin-polarised DOS}

Before we discuss the density of states (DOS) of $h$-BN monolayers that contain substitutional defects, it is important to recall that the pristine $h$-BN monolayer relaxes into the nonmagnetic ground state even when a starting magnetization is imposed. Figure 6 shows the spin-polarised DOS around the band edges within the energy range $\pm 6.0 \mathrm{eV}$ in uncharged $h$-BN monolayers that contain type A (top panels) and type B (bottom panels) complexes. In each case, the Fermi level has been aligned to the $0-\mathrm{eV}$ energy level. Stoichiometric complexes exhibit commensurate DOS in both spin channels by symmetry, and gives rise to zero magnetic moments. By contrast, majority carrier (i.e. spin up channel) DOS and minority carrier (i.e. spin down channel) DOS are non-commensurate in all nonstoichiometric complexes.

The DOS is non-zero at the Fermi level $\left(E_{F}=0 \mathrm{eV}\right)$ in the spin-up channels of the $\mathrm{N}_{1 \mathrm{~B}}+\mathrm{C}_{1 \mathrm{~N}}$ complex, star-shaped C-cluster $\left(\mathrm{C}_{1 \mathrm{~B}}+\mathrm{C}_{3 \mathrm{~N}}\right)$, and the HRC of boron $\left(\mathrm{B}_{3 \mathrm{~N}}\right)$. From the siteresolved ordering of the corresponding spin-polarised charge densities, we conclude that these structures give rise to half-metallic ferromagnetic (HMFM) ground state. The spin down channel in both B- and N- terminated edges show a wide semiconducting band gap. For the star-shaped Ccluster, we only show the DOS of the B-terminated $\left(\mathrm{C}_{1 \mathrm{~B}}+\mathrm{C}_{3 \mathrm{~N}}\right)$ cluster because of its high stability relative to the N-terminated analogue. We find that the TRCs of carbon also give rise to HMFM ground states. Their DOS shows a spontaneous transition from AFM to HMFM ground states when HRCs of carbon are changed to TRCs. We find that embedding either B-terminated (i.e. $\mathrm{C}_{1 \mathrm{~B}}+\mathrm{C}_{3 \mathrm{~N}}$ ) or $\mathrm{N}$-terminated (i.e. $\mathrm{C}_{1 \mathrm{~N}}+\mathrm{C}_{3 \mathrm{~B}}$ ) star-shaped carbon cluster in monolayer $h$ - $\mathrm{BN}$ gives the HMFM ground state. Thus, the half-metallic character of the spin-up channel coupled with the large spin magnetic moment makes the hybrid BCN layer a suitable spin filter. ${ }^{66}$ We also tested for the presence of the non-zero DOS at $E_{F}$ in both B- and N-terminations of larger sizes (i.e. $N_{C}$ $=9$ and 16) of the same defect cluster geometry. Our results show that the carbon-induced HM ferromagnetism is independent of the size or the atomic species at the edge of the embedded cluster. However, when the shape of the cluster is changed to the hexagonal ring geometry, we obtain the AFM ground state for both carbon (i.e. $\mathrm{C}_{3 \mathrm{~B}}+\mathrm{C}_{3 \mathrm{~N}}$ ) and nitrogen (i.e. $\mathrm{N}_{3 \mathrm{~B}}$ ) substitutions. We ascribe the nonmagnetic ground state in the $\mathrm{C}_{3 \mathrm{~B}}+\mathrm{C}_{3 \mathrm{~N}}$ complex to the stoichiometry. Given 
that boron HRC (i.e. $\mathrm{B}_{3 \mathrm{~N}}$ ) also gives a HM ferromagnet, we conclude that the electronic structure in a hybrid BCN monolayer is tunable using the cluster geometry as a degree of freedom.
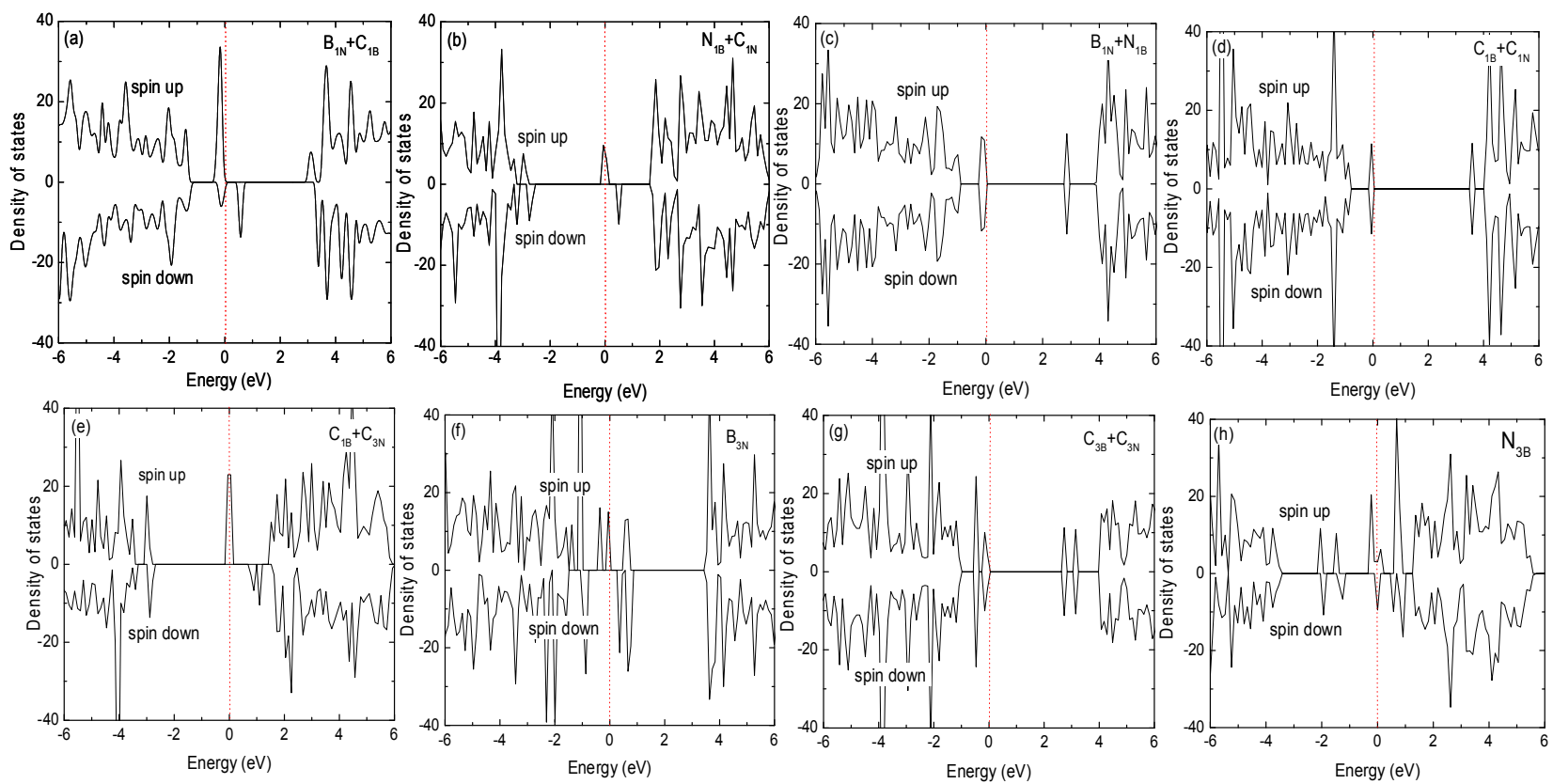

FIG. 6. Electronic DOS around the band edges in type A (top panels) and type B complexes (bottom panels) showing: $\mathrm{B}_{1 \mathrm{~N}}+\mathrm{C}_{1 \mathrm{~B}}(\mathrm{a}), \mathrm{N}_{1 \mathrm{~B}}+\mathrm{C}_{1 \mathrm{~N}}(\mathrm{~b}), \mathrm{B}_{1 \mathrm{~N}}+\mathrm{N}_{1 \mathrm{~B}}(\mathrm{c}), \mathrm{C}_{1 \mathrm{~B}}+\mathrm{C}_{1 \mathrm{~N}}(\mathrm{~d}), \mathrm{C}_{1 \mathrm{~B}}+\mathrm{C}_{3 \mathrm{~N}}(\mathrm{e}), \mathrm{B}_{3 \mathrm{~N}}(\mathrm{f}), \mathrm{C}_{3 \mathrm{~B}}+\mathrm{C}_{3 \mathrm{~N}}$ $(\mathrm{g})$, and $\mathrm{N}_{3 \mathrm{~B}}(\mathrm{~h})$. The Fermi level $\left(E_{F}=0 \mathrm{eV}\right)$ is indicated, in each case, by the dotted vertical line.

\section{Magnetic properties}

\section{Origin of half-metallicity in hybrid BCN monolayers}

The ground state of the stoichiometric double vacancy $\left(\mathrm{V}_{\mathrm{B}}+\mathrm{V}_{\mathrm{N}}\right)$ complex is inherently nonmagnetic. However, when an electron is injected into the layer, it undergoes a transition from the antiferromagnetic (AFM) to half-metallic ferromagnetic (HMFM) ground state, whereas injection of a single hole does not alter the initial nonmagnetic state. ${ }^{67}$ The nonstoichiometric $\mathrm{N}_{1 \mathrm{~B}}+\mathrm{C}_{1 \mathrm{~N}}$ and $\mathrm{B}_{1 \mathrm{~N}}+\mathrm{C}_{1 \mathrm{~B}}$ complexes, for instance, induce spin magnetic moments of 1.01 and 1.06 $\mu_{\mathrm{B}}$, respectively, while stoichiometric $\mathrm{B}_{1 \mathrm{~N}}+\mathrm{N}_{1 \mathrm{~B}}$ and $\mathrm{C}_{1 \mathrm{~B}}+\mathrm{C}_{1 \mathrm{~N}}$ complexes are nonmagnetic. The same trend is observed in nonstoichiometric type $\mathrm{B}$ complexes albeit with larger magnetic moments. The magnetic transitions observed in stoichiometric (or nonstoichiometric) substitutional complexes results in zero (or non-zero) spin polarizations because spin polarization $P=\left[N_{\uparrow}\left(E_{F}\right)-N_{\downarrow}\left(E_{F}\right)\right] /\left(N_{\uparrow}\left(E_{F}\right)+N_{\downarrow}\left(E_{F}\right)\right)$. Here, $N_{\uparrow}\left(E_{F}\right)$ and $N_{\downarrow}\left(E_{F}\right)$ denote the DOS of majority (spin up) and minority (spin down) carriers at the Fermi level. We demonstrate that the halfmetallic (HM) electronic structure in the hybrid $\mathrm{BCN}$ monolayer is an electronic signature of the dominance of majority carriers in bond formation.

Consider that the DOS at $E_{F}$ must only vanish in one spin channel to obtain the HM magnetic ground state. However, in the $\mathrm{B}_{1 \mathrm{~N}}+\mathrm{C}_{1 \mathrm{~B}}$ complex, there is a higher DOS at $E_{F}$ in the spin up channel relative to the spin down channel The difference $N_{\uparrow}\left(E_{F}\right)-N_{\downarrow}\left(E_{F}\right)$, though non-zero, is 
small because of the cancelation effects from electrons with up and down spins. Hence, the observed magnetic moment of $1.06 \mu_{\mathrm{B}}$ suggests a ferromagnetic transition relative to the nonmagnetic ground state of pristine $h$-BN. By contrast, the $0.80 \mu_{\mathrm{B}}$ observed in $\mathrm{N}_{3 \mathrm{~B}}$ complex suggests ferrimagnetism [see Fig. 6(h)]. At zero-bias voltage, the DOS of the hybrid HMFMs clearly favour spin polarised currents, thus making them attractive for spintronic applications. ${ }^{18}$

Due to dangling bonds, open-volume defects introduce electron states, which may either localize as resonances with band edges or as mid-gap states. Also, consider that spin ordering induces non-zero DOS at the Fermi level of an electron-rich $h$-BN monolayer when it contains the divacancy $\left(\mathrm{V}_{\mathrm{N}}+\mathrm{V}_{\mathrm{B}}\right)$ complex. ${ }^{67}$ Because half-metallicity is also observed in some substitutional complexes [see Figs. 6(b), 6(e) and 6(f)], we also investigate the origin of the half-metallic electronic structure. The relaxed geometries of the smallest hexagonal-ring clusters of $\mathrm{C}, \mathrm{N}$ and $\mathrm{B}$ in boronitrene are shown in Fig. 7(a)-(c). Local structure relaxations within each of the hexagonal rings give bond lengths that agree to within 3 sig. figs. The mean length is $1.42 \AA\left(\mathrm{C}_{3 \mathrm{~B}}+\mathrm{C}_{3 \mathrm{~N}}\right)$, $1.43 \AA\left(\mathrm{N}_{3 \mathrm{~B}}\right)$ and $1.53 \AA\left(\mathrm{B}_{3 \mathrm{~N}}\right)$ complexes for $\mathrm{C}-\mathrm{C}, \mathrm{N}-\mathrm{N}$ and $\mathrm{B}-\mathrm{B}$ bonds, in each case. These suggest that locally, i.e., along bonds within the embedded ring, there is no build-up of strain. Thus, there is no frozen-in structural disorder. The $\mathrm{C}_{3 \mathrm{~B}}+\mathrm{C}_{3 \mathrm{~N}}$ and $\mathrm{N}_{3 \mathrm{~B}}$ complexes are nonmagnetic, and give zero magnetic moments whereas strong FM coupling $\left(2.00 \mu_{\mathrm{B}}\right)$ is observed when the boron HRC ( $\mathrm{B}_{3 \mathrm{~N}}$ complex) is embedded in the layer. Similarly, Fig. 7(d) shows the relaxed geometry of the B-terminated star-shaped carbon cluster (i.e. $\mathrm{C}_{1 \mathrm{~B}}+\mathrm{C}_{3 \mathrm{~N}}$ complex).
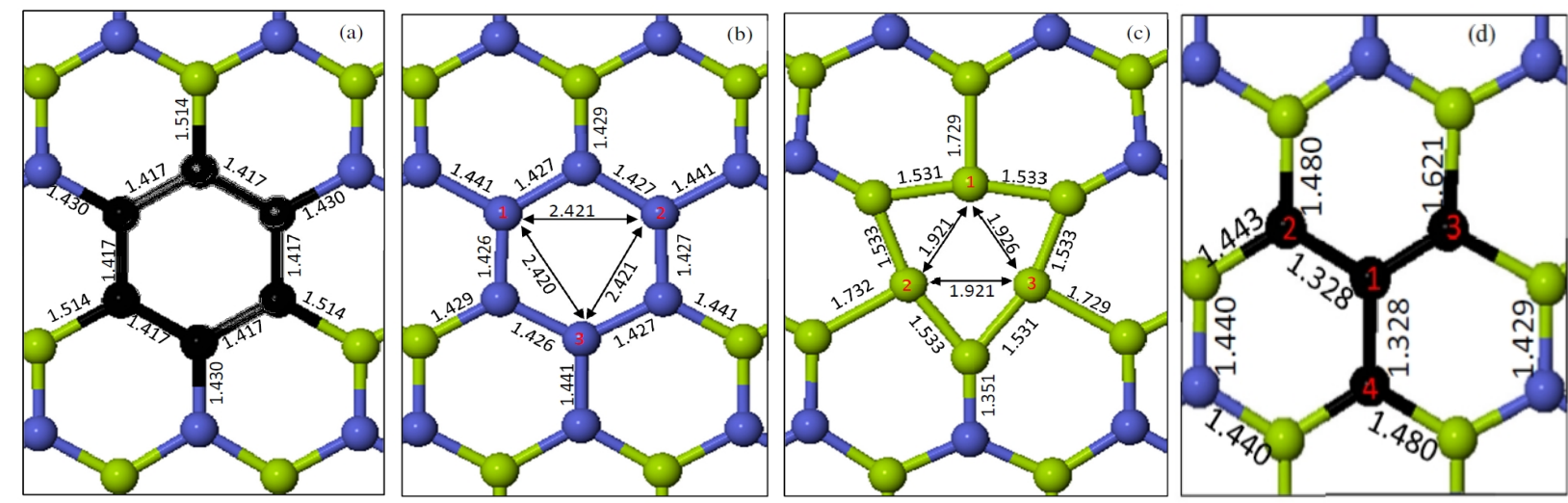

FIG. 7. Relaxed geometries of self-assembled hexagonal ring clusters showing complexes of: C (a), N (b), and B (c). The relaxed local structure of the smallest triangular ring cluster (d). The indicated atomic indices show the substituted $\mathrm{B}$ and $\mathrm{N}$ sublattices of the $\mathrm{N}_{3 \mathrm{~B}}$ and $\mathrm{B}_{3 \mathrm{~N}}$ and $\mathrm{C}_{1 \mathrm{~B}}+\mathrm{C}_{3 \mathrm{~N}}$ complexes, respectively.

Figure 8 shows the angular-momentum-resolved electronic contributions to the total DOS from the four atoms of the smallest TRC of carbon [see Fig. 7(d)]. The $s$ - and $p$-orbital angularmomentum resolved DOS are shown in Fig. 8(a) and (b), respectively. Although the distances (i.e. $1.328 \AA$ ) between the bonded carbon atoms are similar [see Fig. 7(d)], there is considerable asymmetry in the bond lengths around the embedded cluster. In particular, we note that the lengths of the two bonds between boron and carbon $\mathrm{C} 2$ and $\mathrm{C} 4$ are dissimilar. There is a mismatch of $0.037 \AA$ between the bonds, in both cases. An even smaller mismatch of $0.01 \AA$ is also observed between the boron atoms that are bonded to carbon $\mathrm{C} 3$. Around the embedded 
carbon cluster, there are substantial variations, between 1.443 and $1.621 \AA$, in B-C bond lengths. These variations in $\mathrm{B}-\mathrm{C}$ bonds must contribute disproportionate DOS, which contribute to magnetic moments upon spin-splitting. It is important to note that the six B-and three $\mathrm{N}$-atoms of the 12-atom ring that surround the C-cluster [see Fig. 7(d)] contribute non-zero DOS at $E_{F}$. However, their contributions to half-metallicity are minimal compared to those from $\mathrm{C} 2 p$-states.

The atom-resolved s-electron DOS of carbon C1, C2 and C3 show small, but non-zero, density at $\sim-18 \mathrm{eV}$ in the spin-up channel [see arrows in Fig. 8(a)]. There is a high DOS at $\sim-18$ $\mathrm{eV}$ in the spin-up channel of the valence band of the $\mathrm{C}_{1 \mathrm{~B}}+\mathrm{C}_{3 \mathrm{~N}}$ complex, yet carbon $\mathrm{C} 4$ neither contributes $s$-electron states at $-18 \mathrm{eV}$ [see Fig. 8(a)] nor $p$-electron states at $E_{F}$ [see Fig. 8(b)]. In addition, consider that the maximum contribution to the total DOS at $E_{F}$ is 0.0346 and 0.0124 states.eV/cell from $\mathrm{B}$ and $\mathrm{N}$ respectively. These contributions are small compared to the smallest contribution from carbon $\mathrm{C} 4\left(0.0524\right.$ states.eV/cell) in the $\mathrm{C}_{1 \mathrm{~B}}+\mathrm{C}_{3 \mathrm{~N}}$ complex. Since carbon atoms $\mathrm{C} 2$ and $\mathrm{C} 3$ each contribute more than 2.5 states.eV/cell at the Fermi level in the $\mathrm{C}_{1 \mathrm{~B}}+\mathrm{C}_{3 \mathrm{~N}}$ complex, and the spin-up electrons overwhelmingly determine the formation of the B-N bond and its ionicity, we emphasize that the non-zero DOS observed at Fermi level are signatures of the interaction between the itinerant and localised electron states during the hybridization between $\mathrm{C}$ $p$-state and the B $s$-state.
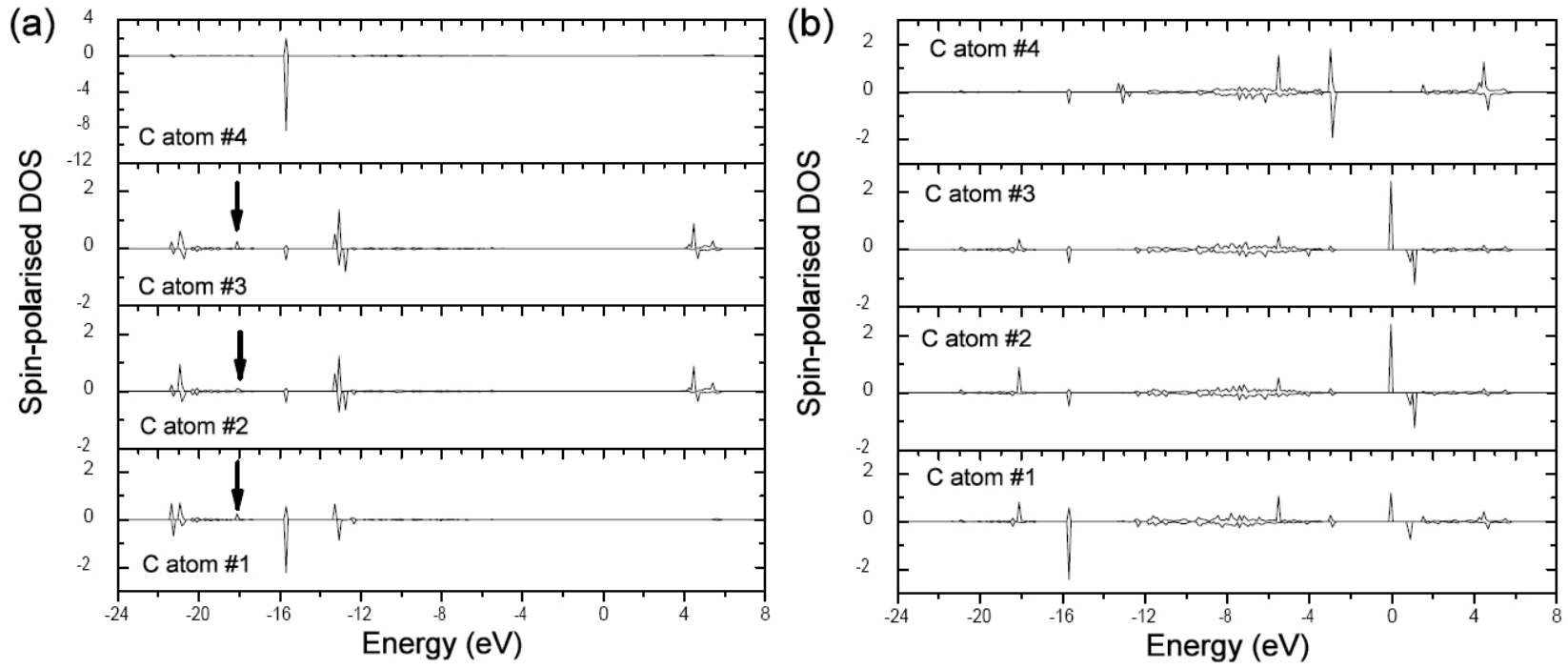

FIG. 8. Spin-polarized electron DOS (in states/eV.supercell) for $s$-orbital (a), and $p$-orbital (b), of the four carbon atoms in the star-shaped carbon cluster. The labels on the atomic contributions correspond to indices shown on the atoms of the smallest TRC of carbon in boronitrene.

\section{Magnetic transitions}

We adopt the Heisenberg model $H=-2 J \sum_{i=1}^{N} \sigma_{i} \sigma_{i+1}$, to interpret the defect-induced magnetic transitions, where $J$ is the exchange coupling and $\sigma_{i}$ denotes the net spin induced by the $i$ th carbon atom. In this case, $\Delta E=E^{A F M}-E^{F M}=2 J\left|\sigma^{2}\right|$. Table II summarises the magnetic transitions due to coupling of spin exchange in neutral hybrid monolayers. For a given configuration, the magnetization energy $\Delta E_{m}$ is evaluated as the total energy difference per 
supercell from nonmagnetic and spin-polarised ground states. Clearly, the AFM ground state has no net magnetic moment although there is spin polarisation (see Figs. 4 and 5). The corresponding FM ground state yields substantially lower total energies, and nonzero magnetization energy, i.e. $\Delta E_{m}>0$, after spin ordering is imposed. The exchange coupling $J$ and the magnetisation energy $\Delta E_{m}$ in ferromagnetically-ordered hybrid $\mathrm{BCN}$ configurations are shown in Table II. This shows that both $J$ and $\Delta E_{m}$ increase monotonically with increasing $N_{C}$. Taken together, we conclude that the magnetic transitions arise from dipole moments from edge-localised defect states which break the symmetry of the electronic structure in both pristine graphene and boronitrene. Finite-size scaling of the ferromagnetic transition as $N_{C} \rightarrow \infty$ should result in sufficiently large magnetic moment to be detected experimentally. ${ }^{68}$

We now consider the possibilities for realizing the half-metallic ferromagnetic ground state with zero magnetization. The magnetization density is the difference charge density per unit volume from spin up and spin down electrons. Fig. 9 shows the $0 \mathrm{~K}$ magnetization density distributions in the identified HM ferromagnets. These show that the magnetization density is strongly localised around defect centres in both type $\mathrm{A}$ and $\mathrm{B}$ complexes. The isosurface plots indicate a maximum density of $0.010,0.076$ and 0.033 e $\AA^{-3}$ in $\mathrm{N}_{1 \mathrm{~B}}+\mathrm{C}_{1 \mathrm{~N}}, \mathrm{~B}_{3 \mathrm{~N}}$ and $\mathrm{C}_{3 \mathrm{~B}}+\mathrm{C}_{6 \mathrm{~N}}$ complex respectively. In Fig. 9(a) and (b), we find that the magnetization density is localised on the $\mathrm{N}$ atoms that surround the embedded complexes, and along the axis of B-C-B bonds. By contrast, it is localised only along B-B bonds of the embedded boron HRC [see Fig. 9(c)]. The magnetic polarization vanishes locally along B-N, N-N, C-C and C-N bonds in all cases [see Fig. 9(a)-(c)]. Thus, we construe the embedded complexes as magnetic domains subject to CurieWeiss law. ${ }^{69}$ The decreased propagation of the magnetization density radially outwards from the centroid of the complexes is attributable to collective spin dynamics ${ }^{70}$ in the HMFMs (see Figs. 4 and 5), in agreement with the interpretation of magnetism as a quantum cooperative effect. ${ }^{71}$

In the following, we show that finite magnetic moments are induced relative to their corresponding ground states when charges are injected into $h$-BN monolayers that contain these complexes. It is clear that the ground state of uncharged $\mathrm{B}_{1 \mathrm{~N}}+\mathrm{N}_{1 \mathrm{~B}}$ and $\mathrm{C}_{1 \mathrm{~B}}+\mathrm{C}_{1 \mathrm{~N}}$ (type $\mathrm{A}$ ) and $\mathrm{N}_{3 \mathrm{~B}}$ and $\mathrm{C}_{3 \mathrm{~B}}+\mathrm{C}_{3 \mathrm{~N}}$ (type $\mathrm{B}$ ) complexes, though magnetically-ordered with zero net magnetic moment (see Table II), is not half-metallic. Fig. 10 shows the modulation of the spontaneous magnetic moment in type A (Fig. 10a) and Type B (Fig. 10b) complexes due to charge injection as predicted using PBE functional. The magnetic transitions obtained using both functionals indicate that the magnetic moments and the type of magnetic transitions in typical $h$-BN hybrid layers are equally tunable using the charge-state of the layer as a degree of freedom. Within the PBE approximation, we find that $\mathrm{B}_{1 \mathrm{~N}}+\mathrm{N}_{1 \mathrm{~B}}$ and $\mathrm{C}_{1 \mathrm{~B}}+\mathrm{C}_{1 \mathrm{~N}}$ complexes possess finite, but variable magnetic moments under charge injection [see Fig. 10(a)]. In the nonstoichiometric $\mathrm{N}_{3 \mathrm{~B}}$ complex for instance, Fig. 10(b) shows that injecting the layer with an electron induces $0.98 \mu_{\mathrm{B}}$. Similarly, injection of a hole also induces the finite magnetic moment of relative to its antiferromagnetic ground state. The stoichiometric $\mathrm{C}_{3 \mathrm{~B}}+\mathrm{C}_{6 \mathrm{~N}}$ complex shows a similar general trend. However, the magnetic moments between $-1 \rightarrow-2$, and $+1 \rightarrow+2$ charge states of the two complexes show opposing characteristics [see Fig. 10(b)]. Nevertheless, further electron (or hole) doping beyond 3 (or +3 ) charge states results in zero magnetic moments. 
Table II. Magnetic exchange coupling and magnetization energy $\Delta E_{m}$ in hybrid BCN monolayers.

\begin{tabular}{cccccc}
\hline \hline Complexes & $N_{C}$ & Spin order & $\Delta E_{m}(\mathrm{eV})$ & $J(\mathrm{eV})$ & $m\left(\mu_{\mathrm{B}}\right)$ \\
\hline $\mathrm{B}_{1 \mathrm{~N}}+\mathrm{N}_{1 \mathrm{~B}}$ & 0 & AFM & 0.00 & & 0.00 \\
$\mathrm{~N}_{1 \mathrm{~B}}+\mathrm{C}_{1 \mathrm{~N}}$ & 1 & HMFM & 0.09 & 0.18 & 1.01 \\
$\mathrm{~B}_{1 \mathrm{~N}}+\mathrm{C}_{1 \mathrm{~B}}$ & 1 & HM & 0.11 & 0.22 & 1.06 \\
$\mathrm{C}_{1 \mathrm{~B}}+\mathrm{C}_{1 \mathrm{~N}}$ & 2 & AFM & 0.00 & & 0.00 \\
$\mathrm{~B}_{3 \mathrm{~N}}$ & 0 & HM & 0.21 & 0.42 & 1.99 \\
$\mathrm{~N}_{3 \mathrm{~B}}$ & 0 & HMFM & 0.00 & & 0.00 \\
$\mathrm{C}_{1 \mathrm{~B}}+\mathrm{C}_{3 \mathrm{~N}}$ (or $\left.\mathrm{C}_{1 \mathrm{~N}}+\mathrm{C}_{3 \mathrm{~B}}\right)$ & 4 & HMFM & 0.33 & 0.66 & $2.00(1.99)$ \\
$\mathrm{C}_{3 \mathrm{~B}}+\mathrm{C}_{3 \mathrm{~N}}$ & 6 & AFM & 0.00 & & 0.00 \\
$\mathrm{C}_{3 \mathrm{~B}}+\mathrm{C}_{6 \mathrm{~N}}$ (or $\left.\mathrm{C}_{6 \mathrm{~B}}+\mathrm{C}_{3 \mathrm{~N}}\right)$ & 9 & HMFM & 0.72 & 1.42 & $3.14(3.04)$ \\
$\mathrm{C}_{6 \mathrm{~B}}+\mathrm{C}_{10 \mathrm{~N}}\left(\right.$ or $\left.\mathrm{C}_{6 \mathrm{~N}}+\mathrm{C}_{10 \mathrm{~B}}\right)$ & 16 & HMFM & 0.90 & 1.80 & $3.77(3.33)$ \\
\hline
\end{tabular}
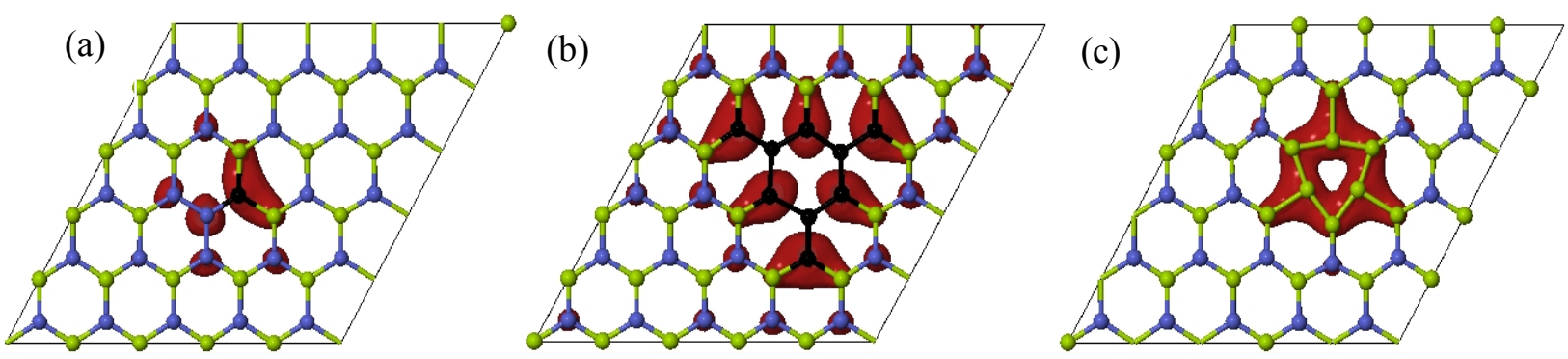

FIG. 9: Isosurface maps of the $0 \mathrm{~K}$ magnetization densities in half-metallic ferromagnets from: $\mathrm{N}_{1 \mathrm{~B}}+\mathrm{C}_{1 \mathrm{~N}}(\mathrm{a}), \mathrm{B}_{3 \mathrm{~N}}(\mathrm{~b})$, and $\mathrm{C}_{3 \mathrm{~B}}+\mathrm{C}_{6 \mathrm{~N}}(\mathrm{c})$ complexes.
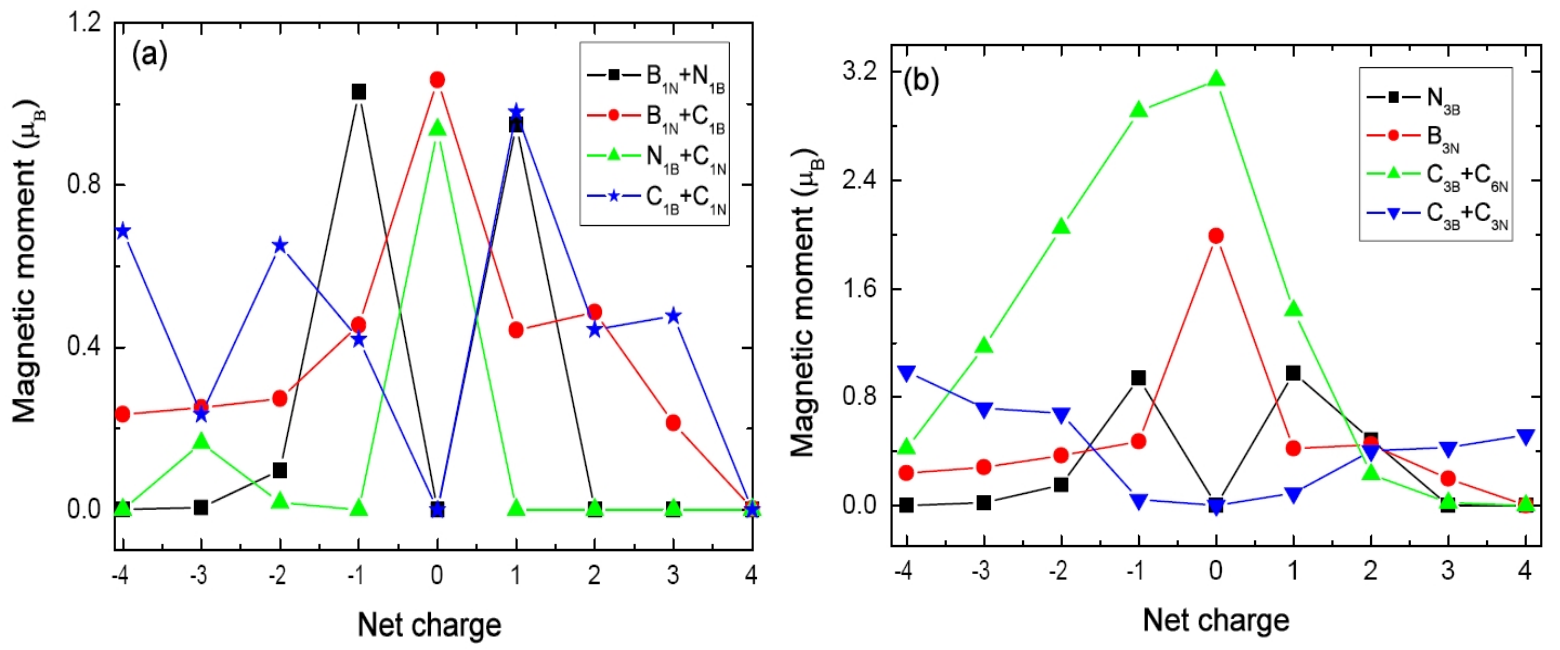

FIG.10. Charge-tunable magnetism in substitutionally-doped boronitrene layers.

Taking into consideration the known deficiencies of the PBE functional approximation in predicting the electronic structure of boronitrene, ${ }^{52,55}$ we also investigate the charge-tunability of the defect-induced magnetism in the prototypical $\mathrm{C}_{1 \mathrm{~B}}+\mathrm{C}_{3 \mathrm{~N}}$ complex, under the effect of quasiparticle-corrections using the HSE functional, and compare the results with similar PBE 
calculations. From PBE and HSE06 functional calculations, we find a consistent trend for strong dependence of the induced magnetic moment on levels of charge injection. Both functionals predict slightly different absolute values but maximal magnetic moment in the uncharged state. For instance, PBE gives exactly $2.00 \mu_{\mathrm{B}}$ (see Table II) while HSE06 yields marginally higher magnetic moment of $2.034 \mu_{\mathrm{B}}$ in the 0 charge state. Both functionals also give consistent trends in the + and - charge states of the complex. In both -1 and +1 charge states, the PBE saturation moment reduces to 1.03 and $1.00 \mu_{\mathrm{B}}$, while the HSE06 functional predicts corresponding moments of 1.06 and $1.02 \mu_{\mathrm{B}}$. In both cases, we also find a vanishing magnetic moment at the -2 charge state. Injection of an electron for the -2 to -3 transition switches the magnetic moment from 0 to $1.00 \mu_{\mathrm{B}}$. By contrast, charge injection beyond +1 up to +4 charge states gives zero net magnetic moment using both functionals. Thus, although large quasiparticle corrections influence the size of the band gap in boronitrene, such corrections have minimal effects on the carbonimpurity induced electronic states that are localised at the Fermi level.

From Fig. 2, it is clear that a continuous increase in the magnitude of the injected positive charges results in increased thermodynamic instability in defective $h$-BN monolayers. Nevertheless, the $\mathrm{B}_{3 \mathrm{~N}}$ complex is somewhat more stable relative to other complexes. The instability suggests that the electronic structure has been driven away from the equilibrium conditions in the high hole-doping regime. The driven transition to thermodynamic instability regimes must therefore be responsible for the observed switching-off of the magnetic moments in both types $\mathrm{A}$ and $\mathrm{B}$ complexes. We therefore suggest that heavy hole-doping of $h$-BN nanostructures is a useful way to switch off the magnetic moments, and therefore control the magnetism in $h$-BN nanostructures. Taken together, we conclude that the site ordering and the geometry of the embedded complexes determine the magnetic transitions, which are tunable by charge injection. This shows that the magnetic moment due to the HM ferromagnetism of the uncharged $\mathrm{C}_{1 \mathrm{~B}}+\mathrm{C}_{3 \mathrm{~N}}$ complex [see Fig. 6(e)] decreases to give $\mathrm{HM}$ ferrimagnetism when electrons (i.e. up to -4), and holes (i.e. up to +2 ), are injected into the layer. For the half-metallic $\mathrm{B}_{3 \mathrm{~N}}$ complex, the same trend is obtained. However, the magnetic moment is only quenched when the injected charge is +4 . Because the electronic DOS at the Fermi level must vanish only in one spin channel to obtain the HM magnetic ground state, we conclude that the half-metallic electronic structure is tunable.

Figure 11 shows the response of the magnetic moment in $\mathrm{HM}$ ferromagnets to thermal excitation. The charge-modulation of the effect of heat on the magnetic moment in the $\mathrm{N}_{1 \mathrm{~B}}+\mathrm{C}_{1 \mathrm{~B}}$ complex is shown in Fig. 11(a). The effect of cluster size on the magnetic moment is shown in Fig. 11(b). The response of the magnetic moment to temperature in three different sizes of graphene flakes $\left(4 \leq N_{C} \leq 16\right)$ is shown because of their high thermodynamic stability relative to the $\mathrm{B}_{3 \mathrm{~N}}$ complex. The macroscopic magnetic moments in HMFMs (see Table II) undergo a transition to HM ferrimagnetism as a response to heat. The magnetic moments reduce abruptly above $750 \mathrm{~K}$ in all cases, and vanish beyond $1250 \mathrm{~K}$ in carbon substitutional complexes independent of $N_{C}$ in line with Curie-Weiss law. Contrary to a priori expectations for temperature mediated quenching of magnetic moments, we find a significantly different response to heat when the $\mathrm{B}_{3 \mathrm{~N}}$ complex is embedded in the $h$-BN monolayer. The $1.99 \mu_{\mathrm{B}}$ magnetic moment at $0 \mathrm{~K}$ is unchanged at $3500 \mathrm{~K}$. Because of the onset of melting at $3450 \mathrm{~K}$ - as seen in the pair correlation function - we equilibrated the $\mathrm{B}_{3 \mathrm{~N}}$ hybrid monolayer for a further $100 \mathrm{ps}$ at its melting point (i.e. $3500 \mathrm{~K})$. Quite unusually, the $3500 \mathrm{~K}$ magnetic moment $\left(1.9889 \mu_{\mathrm{B}}\right)$ shows very negligible 
changes from the $0 \mathrm{~K}\left(1.99 \mu_{\mathrm{B}}\right)$ value. The ordered structure of the $h$-BN monolayer is lost at $3450 \mathrm{~K}$ due to structural disorder at the melting point - without any appreciable change in the macroscopic magnetic moment. Therefore, we conclude that impurity-free boronitrene gives an ultra-high temperature half-metallic ferromagnet when any three nearest neighbour $\mathrm{N}$ atoms are replaced with $B$.
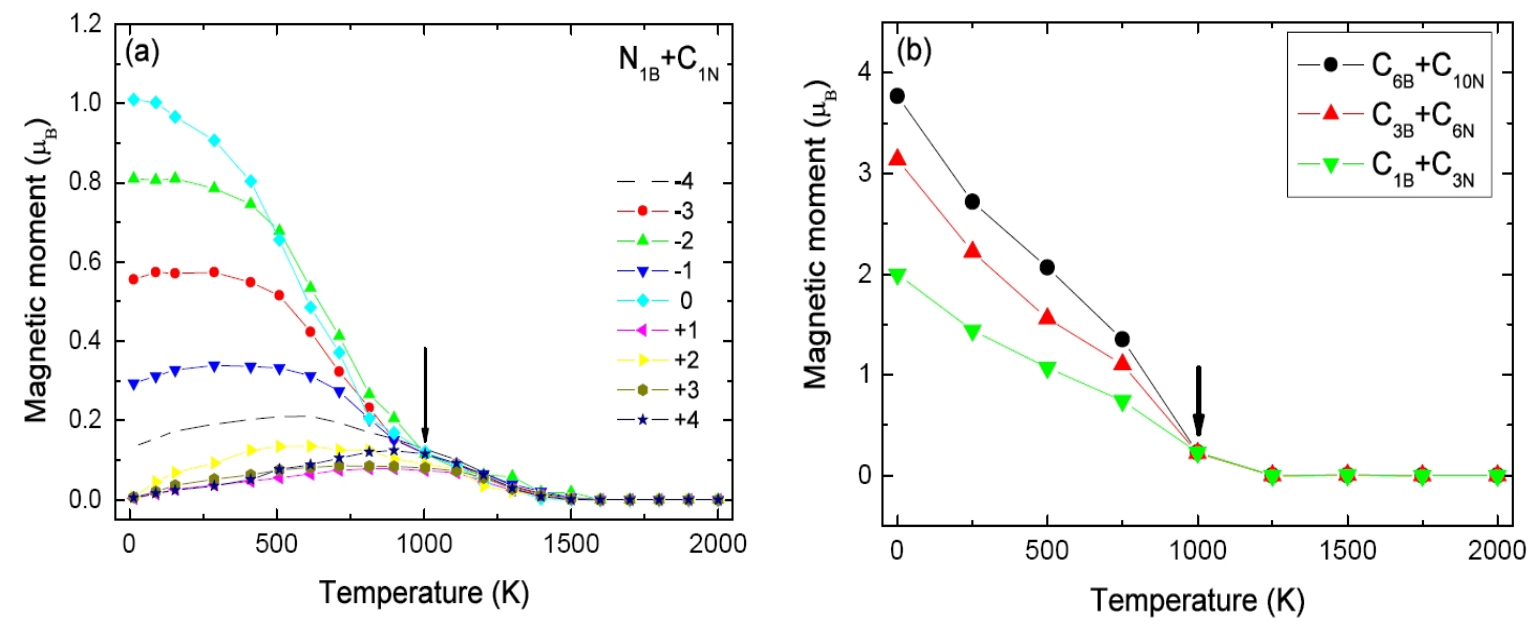

FIG. 11. Temperature-tunable half-metallic ferromagnetism: effects of charge modulation in the $\mathrm{N}_{1 \mathrm{~B}}+\mathrm{C}_{1 \mathrm{~B}}$ complex (a), and effect of cluster size in the substitutional carbon complexes (b).

\section{CONCLUSIONS}

We have presented the results of first principles molecular dynamics simulation of the response of the magnetic and electronic properties of substitutionally-doped $h$-BN monolayers to finite temperature. The possible pathways for realizing the half-metallic ferrimagnetic ground state in $h$-BN nanostructures, with zero magnetization, has been explored. We find that boronterminated carbon clusters are relatively more stable in boron-rich conditions than nitrogenterminated carbon clusters in nitrogen-rich conditions. Our results suggest that triangular graphene flakes induce half-metallic ferromagnetism when embedded in boronitrene. By contrast, hexagonal graphene flakes are nonmagnetic. It is found that the nonmagnetic ground state in boronitrene can be tuned to give half-metallic ferromagnetism by embedding clusters of carbon in triangular geometry. In addition, we find that magnetism in the hybrid monolayers is equally tunable by charge modulation. The induced half-metallic ferromagnetism survives up to the melting point in carbon-free boronitrene when three nearby nitrogen atoms are substituted with three boron atoms.

\section{ACKNOWLEDGEMENT}

AMU thanks the University of Pretoria (UP) for financial support under Grant E2020(5). NC thanks the University of Pretoria and the National Institute for Theoretical Physics for support. We are grateful to Prof. Jannie Pretorius for computational support on the UP clusters. 


\section{REFERENCES}

${ }^{1}$ S. P. Dash, S. Sharma, R. S. Patel, M. P. de Jong, and R. Jansen, Nature 462, 491 (2009).

${ }^{2}$ M. Tran, H. Jaffrès, C. Deranlot, J.-M. George, A. Fert, A. Miard, and A. Lemaître, Phys. Rev. Lett.

102, 036601 (2009).

${ }^{3}$ M. Ciorga, A. Einwanger, U. Wurstbauer, D. Schuh, W. Wegscheider, and D. Weiss, Phys. Rev. B

79, 165321 (2009).

${ }^{4}$ I. Appelbaum, B. Huang, and D. J. Monsma, Nature 447, 295 (2007).

${ }^{5}$ J. Flipse, F. L. Bakker, A. Slachter, F. K. Dejene, and B. J. van Wees, Nature Nanotech. 7, 166 (2012).

${ }^{6}$ G. E. W. Bauer, E. Saitoh, and B. J. van Wees, Nature Mater. 11, 391(2012).

${ }^{7}$ T. L. Makarova, B. Sundqvist, R. Hohne, P. Esquinazi, K. Kopelevich, P. Scharff, V. A. Davydov, L.

A. Kahsevarova, and A. V. Rakhmanina, Nature 413, 716 ( 2001).

${ }^{8}$ P. Esquinazi, A. Setzer, R. Höhne, C. Semmelhack, Y. Kopelevich, D. Spemann, T. Butz, B.

Kohlstrunk, and M. Lösche, Phys. Rev. B 66, 024429 (2002).

${ }^{9}$ Y. Shibayama, H. Sato, T. Enoki, and M. Endo, Phys. Rev. Lett. 84, 1744 (2000).

${ }^{10}$ M. Fujita, K.Wakabayashi, K. Nakada, and K. Kusakabe, J. Phys. Soc. Jpn. 65, 1920 (1996).

${ }^{11}$ K. Nakada, M. Fujita, G. Dresselhaus, and M. S. Dresselhaus, Phys. Rev. B 54, 17954 (1996).

${ }^{12}$ A. N. Andriotis, M. Menon, R.M. Sheetz, and L. Chernozatonskii, Phys. Rev. Lett. 90, 026801 (2003).

${ }^{13}$ K. Kusakabe and M. Maruyama, Phys. Rev. B 67, 092406 (2003).

${ }^{14}$ Y. H. Kim, J. Choi, K. J. Chang, and D. Tománek, Phys. Rev. B 68125420 (2003).

${ }^{15}$ D.V. Khveshchenko, Phys. Rev. Lett. 87, 206401 (2001).

${ }^{16}$ D.V. Khveshchenko, Phys. Rev. Lett. 87, 246802 (2001).

${ }^{17}$ N. Park, M. Yoon, S. Berber, J. Ihm, E. Osawa, and D. Tománek, Phys. Rev. Lett. 91, 237204 (2003).

${ }^{18}$ I. Zütić, J. Fabian and S. Das Sarma, Rev. Mod. Phys. 76, 323 (2004).

${ }^{19}$ J. Bai, R.Cheng, F. Xiu, L. Liao, M. Wang, A. Shailos, K. L. Wang, Y. Huang, and X. Duan, Nature Nanotech. 5, 655 (2010).

${ }^{20}$ K. S. Novoselov, D. Jiang, F. Schedin, T. J. Booth, T. T. Khotkevich, S. V. Morozov, and A. K. Geim, Proc. Natl. Acad. Sci. USA 102, 10451 (2005).

${ }^{21}$ K. S. Novoselov, A. K Geim, S. V. Morozov, D. Jiang, Y. Zhang, S. V. Dubonos, I. V. Grigorieva, and A. A. Firsov, Science 306, 666 (2004).

${ }^{22}$ L. Ci, L. Song, C. Jin, D. Jariwala, D. Wu, Y. Li, A. Srivastava, Z. F. Wang, K. Storr, L. Balicas, F. Liu, and P. M. Ajayan, Nature Mater. 9, 430 (2010).

${ }^{23}$ A. Rubio, Nature Mater. 9, 379 (2010).

${ }^{24}$ A. L. M. Reddy, A. Srivastava, S. R. Gowda, H. Gullapalli, M. Dubey, and P. M. Ajayan, ACS Nano 4, 6337 (2010).

${ }^{25}$ X. Wang, X. Li, L. Zhang, Y. Yoon, P. K. Weber, H. Wang, J. Guo, and H. Dai Science 324, 768 (2009).

${ }^{26}$ J. C. Meyer S. Kurasch, H. J. Park, V. Skakalova, D. Künzel, A. Groß, A. Chuvilin, G. Algara-Siller, S. Roth, T. Iwasaki, U. Starke, J. H. Smet, and U. Kaiser, Nature Mater. 10, 209 (2011).

${ }^{27}$ E.-J. Kan, X. Wu, Z. Li, X. C. Zeng, J.Yang, and J. G. Hou, J. Chem. Phys. 129, 084712 (2008).

${ }^{28}$ A. V. Krasheninnikov, P.O. Lehtinen, A.S. Foster, P. Pyykko, and R. M. Nieminen, Phys. Rev. Lett. 102,126807 (2009).

${ }^{29}$ Y. Ma, Y. Dai, M. Guo, C. Niu, L. Yu, and B. Hunag, Nanoscale 3, 2301 (2011).

${ }^{30}$ J. S. Crvenka, M. I. Katsnelson, and C. F. J. Flipse, Nature Phys. 5, 840 (2009).

${ }^{31}$ R. F. Liu and C. Cheng, Phys. Rev. B 76, 014405 (2007).

${ }^{32}$ M. S. Si and D. S. Xue, Phys. Rev. B 75, 193409 (2007).

${ }^{33}$ J. M. Pruneda, Phys. Rev. B 85, 045422 (2012).

${ }^{34}$ O. L. Krivanek, M. F. Chisholm, V. Nicolosi, T. J. Pennycook, G. J. Corbin, N. Dellby, M. F. Murfitt, C. S. Own, Z. S. Szilagyi, M. P. Oxley, S. T. Pantelides and S. J. Pennycook, Nature (London) 464, 571 (2010).

${ }^{35}$ X. Wei, M. Wang, Y. Bando, and D. Golberg, J. Am.Chem. Soc. 132, 13592 (2010).

${ }^{36}$ X.Wei, M. Wang, Y. Bando, and D. Golberg, ACS Nano 5, 2916 (2011). 
${ }^{37}$ N. Berseneva, A. V. Krasheninnikov, and R. M. Nieminen, Phys. Rev. Lett. 107, 035501(2011).

${ }^{38}$ Z. M. Liu, Y. Zhu, and Z. Q. Yang, J. Chem. Phys. 134, 074708 (2011).

${ }^{39}$ S. Okada, M. Igami, K. Nakada, and A. Oshiyama, Phys. Rev. B 62, 9896 (2000).

${ }^{40}$ Y.-W. Son, M. L. Cohen, and S. G. Louie, Nature (London) 444, 347 (2006).

${ }^{41}$ S. Dutta, A. K. Manna, and S. K. Patti, Phys. Rev. Lett. 102, 096601 (2009).

${ }^{42}$ G. Kresse and J. Hafner Phys. Rev. B 47, 558 (1993).

${ }^{43}$ G. Kresse and J. Hafner Phys. Rev. B 49, 14251 (1994).

${ }^{44}$ G. Kresse and J. Furthmuller, Phys. Rev. B 54, 11169 (1996).

${ }^{45}$ G. Kresse and J. Furthmuller, Comput. Mater. Sci. 6, 11169 (1996).

${ }^{46}$ MedeA Materials Exploration and Design Analysis Software (Materials Design, Inc.) v 2.10. Available online at www.materialsdesign.com/medea.

${ }^{47}$ J. P. Perdew, K. Burke, and M. Ernzerhof, Phys. Rev. Lett. 77, 18 (1996).

${ }^{48}$ G. Kresse and D. Joubert, Phys. Rev. B 59, 1758 (1999).

${ }^{49}$ P. E. Blöchl, Phys. Rev. B 50, 17953 (1994).

${ }^{50}$ M. Methfessel and A. T. Paxton, Phys. Rev. B 40, 3616 (1989).

${ }^{51}$ H. J. Monkhorst and J. D. Pack, Phys. Rev. B 13, 5188 (1976).

${ }^{52}$ T. B. Ngwenya, A. M. Ukpong, and N. Chetty, Phys. Rev. B 84, 245425 (2011).

${ }^{53}$ V. Wang, N. Ma, H. Mizuseki, and Y. Kawazoe, Solid State Commun. 152, 816 (2012).

${ }^{54}$ S. B. Zhang and J. E. Northrup, Phys. Rev. Lett. 67, 2339 (1991).

${ }^{55}$ C. Attaccalite, M. Bockstedte, A. Marini, A. Rubio, and L. Wirtz, Phys. Rev. B 83, 144115 (2011).

${ }^{56}$ J. Heyd, G. E. Scuseria, and M. Ernzerhof, J. Chem. Phys. 118, 8207 (2003).

${ }^{57}$ J. Heyd, G. E. Scuseria, and M. Ernzerhof, J. Chem. Phys. 124, 9906 (2006).

${ }^{58}$ V. L. Solozhenko and V. Turkevich, J. Phys. Chem. B 103, 2903 (1999).

${ }^{59}$ A. M. Ukpong, Mol. Phys. 107, 2521 (2009).

${ }^{60}$ S. Lany and A. Zunger, Phys. Rev. B 78, 235104 (2008).

${ }^{61}$ J. Shim, E.-K. Lee, Y. J. Lee, and R. M. Nieminen, Phys. Rev. B 71, 245204 (2005).

${ }^{62}$ J. Lento, J. -L. Mozos, and R. M. Nieminen, J. Phys. Condens. Matter 14, 2637 (2002).

${ }^{63}$ G. Makov and M. C. Payne, Phys. Rev. B 51, 4014 (1995).

${ }^{64}$ M. I. J. Probert and M. C. Payne, Phys. Rev. B 67, 075204 (2003).

${ }^{65}$ C. G. Van de Walle and J. Neugenbauer, J. Appl. Phys. 95, 3851 (2004).

${ }^{66}$ P. Michetti and P. Recher, Phys. Rev. B 84, 125438 (2011).

${ }^{67}$ A. M. Ukpong and N. Chetty, J. Phys. Condens. Matter 24, 65002 (2012).

${ }^{68}$ M. Kan, J. Zhou, Q. Sun, Q. Wang, Y. Kawazoe, and P. Jena, Phys. Rev. B 85, 155450 (2012).

${ }^{69} \mathrm{C}$. Kittel, Introduction to Solid State Physics (John Wiley, New Jersey, 2005).

${ }^{70}$ V. K. Henner, V. I. Yukalov, P. V. Kharebov, and E. P. Yukalova, J. Phys. Conf. Ser. 129, 012015 (2008).

${ }^{71}$ A. L. Kuzemsky, Physics of Particles and Nuclei 40, 949 (2009). 